\title{
Unravelling the Molecular Origin of the Rigiospecificity in Extradiol Catechol Dioxygenases
}

\author{
Gemma J. Christian, ${ }^{1,2}$ Frank Neese ${ }^{1}$ and Shengfa $\mathrm{Ye}^{1}$
}

${ }^{1}$ Max-Planck Institute for Chemical Energy Conversion, Stiftstr. 34-36, D-45470

Mülheim an der Ruhr, Germany

${ }^{2}$ Avondale College of Higher Education, Cooranbong, New South Wales 2265, Australia

\section{Supplementary Information}

1 Validation of the computational setup ............................................................. 2

2 Evidence for the oxidation of the substrate to a quinone .............................. 4

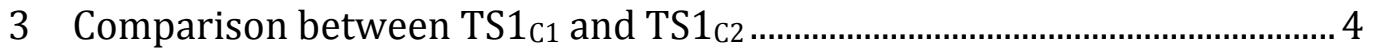

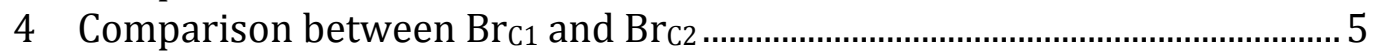

5 Comparison of the geometries and electronic structures of TS2 $2_{\mathrm{C} 1}$ and

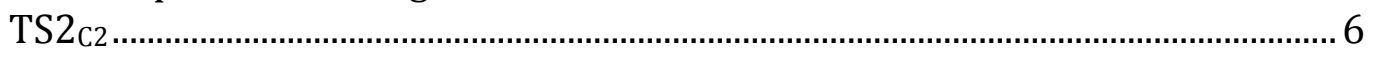

6 The structure of the quasi- intermediate............................................................. 7

7 Relationship between orbital energies and the barrier to intradiol

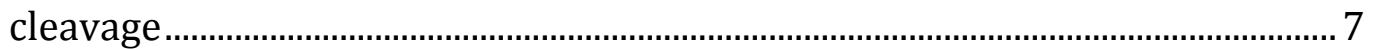

8 Cartesion coordinates of the calculated structures of key species........... 10 


\section{Validation of the computational setup}

Table S1. Comparison of optimized geometries and spin populations with and without the relativistic ZORA corrections for the quintet $\mathrm{O}_{2}$ adduct.

\begin{tabular}{|l|l|l|}
\hline \multicolumn{2}{|l|}{ ZORA-B3LYP } & \multicolumn{1}{l|}{ B3LYP } \\
\hline \multicolumn{2}{|l|}{ Key structure parameters } & \multicolumn{2}{l|}{} \\
\hline Fe- $\mathrm{O}_{\mathrm{C} 2}(\AA)$ & 1.995 & 2.044 \\
\hline Fe- $\mathrm{C}_{\mathrm{C} 1}(\AA)$ & 1.978 & 2.023 \\
\hline Fe-01 $(\AA)$ & 2.242 & 2.269 \\
\hline $01-02(\AA)$ & 1.326 & 1.336 \\
\hline $\mathrm{C} 2-\mathrm{O}_{\mathrm{C} 2}(\AA)$ & 1.341 & 1.320 \\
\hline $\mathrm{C} 1-\mathrm{O}_{\mathrm{C} 1}(\AA)$ & 1.335 & 1.315 \\
\hline $\mathrm{C} 1-\mathrm{C} 2(\AA)$ & 1.429 & 1.441 \\
\hline Spin population & & \\
\hline $\mathrm{C}_{01}$ & $0.18(0.19)$ & $0.20(0.21)$ \\
\hline $\mathrm{C}_{02}$ & $0.19(0.19)$ & $0.20(0.21)$ \\
\hline$\Sigma C_{\text {ring }}$ & $0.088(0.085)$ & $0.27(0.23)$ \\
\hline Sub & $0.45(0.46)$ & $0.68(0.66)$ \\
\hline 01 & $-0.59(-0.57)$ & $-0.55(-0.54)$ \\
\hline 02 & $-0.33(-0.33)$ & $-0.34(-0.34)$ \\
\hline
\end{tabular}

*The values from the Mullikin analysis are listed and those from the Löwdin analysis are put in paratheses. 


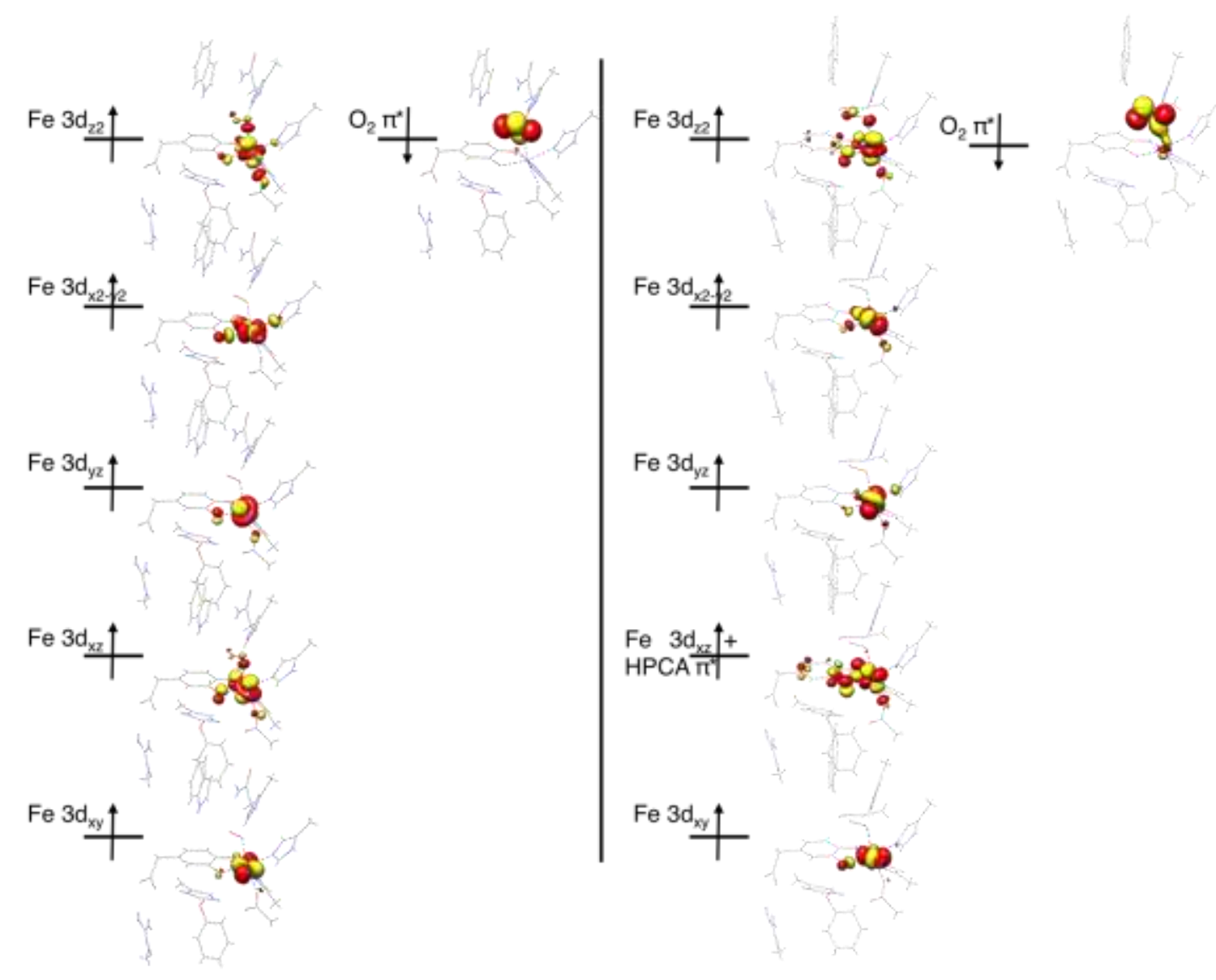

Figure S1 Schematic MO diagram for the $\mathrm{O}_{2}$ adduct computed with ZORA approximation (left) and without it (right) . Unrestricted corresponding orbitals are used to represent the spin-coupled pair and the remaining orbitals are quasi-restricted orbitals. 


\section{Evidence for the oxidation of the substrate to a quinone}

Selected bond lengths are tabulated in Table 1 . There is a noticeable shortening of the $\mathrm{C}_{1}-\mathrm{O}_{\mathrm{C} 1}$ and $\mathrm{C}_{2}-\mathrm{O}_{\mathrm{C} 2}$ bond lengths from the superoxide to just after TS1, consistent with the formation of a $\mathrm{C}=\mathrm{O}$ double bond. Once the superoxide has attacked the substrate to form the new $\mathrm{O}_{2}-\mathrm{C}_{2}$ bond the $\mathrm{C}_{2}-\mathrm{O}_{\mathrm{C} 2}$ lengthens, consistent with the nucleophilic attack of the $\mathrm{C}=0 \mathrm{\pi}$ bond.s

Table S2. Selected calculated bond lengths $(\AA)$.

\begin{tabular}{|l|c|c|c|}
\hline & Superoxide & Just after TS1 & Br \\
\hline $\mathrm{O}_{2}-\mathrm{C}_{2}$ & - & 2.1 & 1.507 \\
\hline $\mathrm{C}_{2}-\mathrm{O}_{\mathrm{C} 2}$ & 1.397 & 1.279 & 1.345 \\
\hline $\mathrm{C}_{1}-\mathrm{O}_{\mathrm{C} 1}$ & 1.400 & 1.255 & 1.240 \\
\hline
\end{tabular}

3 Comparison between $\mathrm{TS} 1_{\mathrm{C} 1}$ and $\mathrm{TS} 1_{\mathrm{C} 2}$

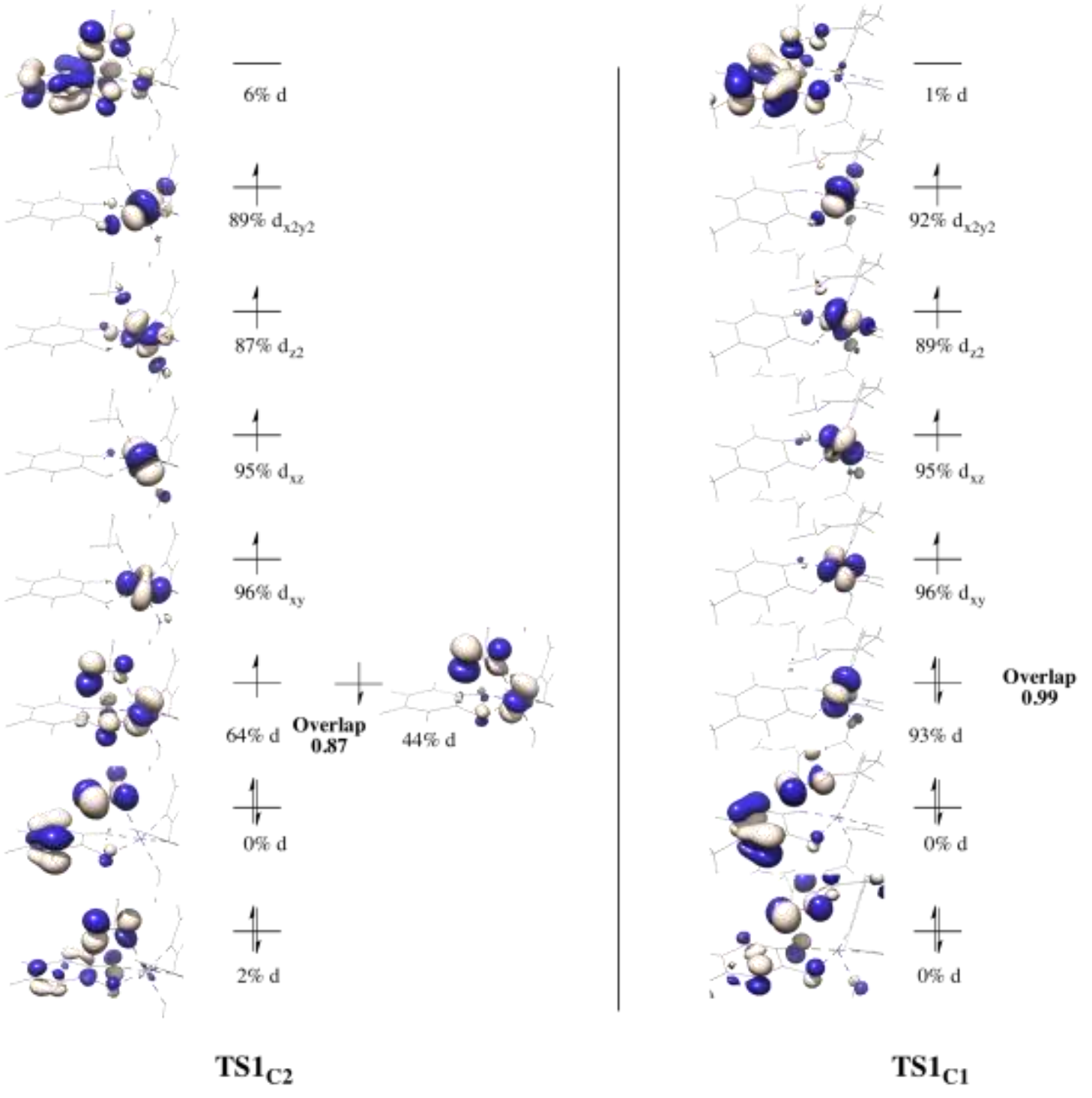


Figure S2 Molecular orbital diagrams for $\mathrm{TS} 1_{\mathrm{C} 1}$ and $\mathrm{TS} 1_{\mathrm{C} 2}$. Unrestricted corresponding orbitals are used to represent the spin-coupled pair (overlap in bold) and all other orbitals are quasi-restricted orbitals.

\section{Comparison between $\mathrm{Br}_{\mathrm{C} 1}$ and $\mathrm{Br}_{\mathrm{C} 2}$}

Apart from the obvious differences in the position of attack on the substrate, the structure for $\mathrm{Br}_{\mathrm{C} 1}$ shows increased distortion from linearity in the hydrogen bond between His200 and $\mathrm{O}_{1}$, with a change from an $\mathrm{O}_{1}-\mathrm{H} . . . \mathrm{N}_{\mathrm{His} 200}$ angle of $176^{\circ}$ for $\mathrm{Br}_{\mathrm{C} 2}$ to $168^{\circ}$ for $\mathrm{Br}_{\mathrm{C} 1}$. The distortion suggests that the active site may impose geometric constraints on the peroxo bridge.

To test this hypothesis, the constraints on the truncating atom on His200 were released. This does not lead to a dramatic effect on the selectivity, with $\mathrm{Br}_{\mathrm{C} 2}$ still $26.9 \mathrm{~kJ} \mathrm{~mol}^{-1}$ below $\mathrm{Br}_{\mathrm{C} 1}$ in energy. Furthermore only slight changes in the position of His200 and the hydrogen bond with the hydroperoxo bridge were observed. This was initially puzzling, however closer inspection of the structures shows that His200 lies close to the neighboring His155, which has the potential to restrict its movement. The distance between the nitrogen donor atoms on these two residues is $3.377 \AA$ for $\mathrm{Br}_{\mathrm{C} 2}$ and $3.509 \AA$ for $\mathrm{Br}_{\mathrm{C} 1}$ indicating potential crowding.

Release of the constraints on both His155 and His200 has a significant effect on not only the relative energy of $\mathrm{Br}_{\mathrm{C} 1}$ and $\mathrm{Br}_{\mathrm{C} 2}$ but also their geometries. Shorter Fe-N(His) bonds were calculated for both complexes but for $\mathrm{Br}_{\mathrm{C} 1}$ significant rotation of His200 and His155 was also observed, allowing opening of the $\mathrm{O}_{2}$ $\mathrm{H} . . . \mathrm{N}_{\mathrm{His} 200}$ angle to $178^{\circ}$. Importantly, after the release of these constraints $\mathrm{Br}_{\mathrm{C} 2}$ is $14.5 \mathrm{~kJ} \mathrm{~mol}^{-1}$ lower in energy than $\mathrm{Br}_{\mathrm{C} 1}$, i.e. a change of $13.9 \mathrm{~kJ} \mathrm{~mol}^{-1}$ compared to the native system.

Further calculations demonstrate that the constraints on His155 are only significant when His200 is present, and that releasing the constraints on His155 while maintaining those on His200 do not exert a dramatic effect on the selectivity. Therefore this is a concerted effect, where the combination of the constraints on His200 and His155 lead to distortion of $\mathrm{Br}_{\mathrm{C} 1}$. Selectively releasing constraints on other residues such as the salt bridge, showed only minor changes. 

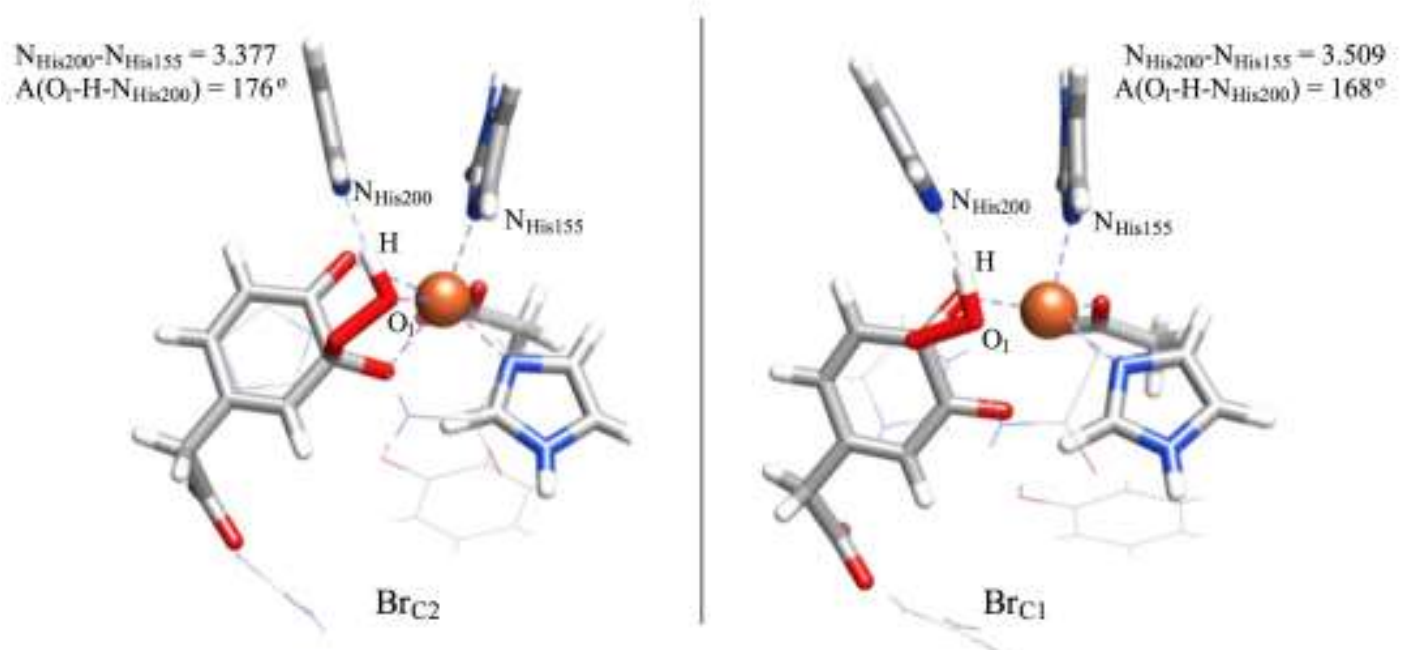

Figure S3 Distortion in the structure of the peroxo bridge for $\mathrm{Br}_{\mathrm{C} 2}$. Second sphere residues are shown as a wire-frame.

5 Comparison of the geometries and electronic structures of TS2 $2_{\mathrm{C} 1}$ and TS2

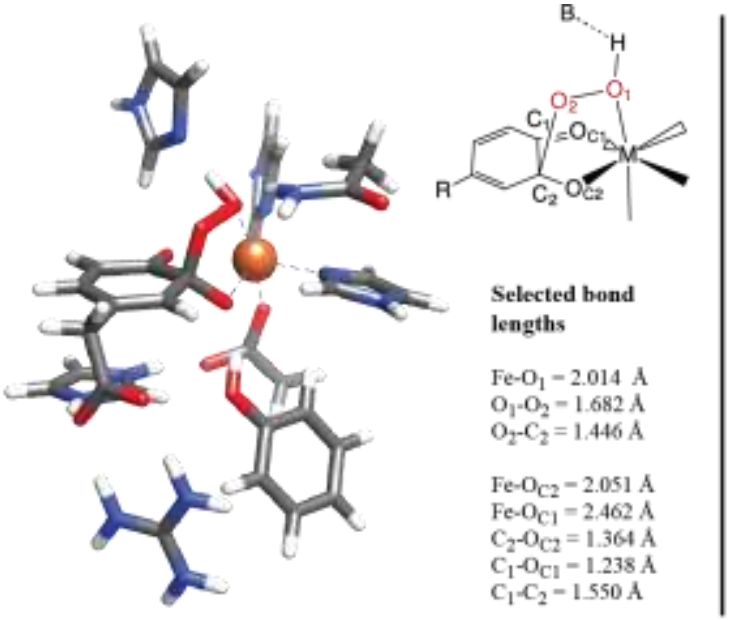

TS2 $_{\mathrm{C} 2}$

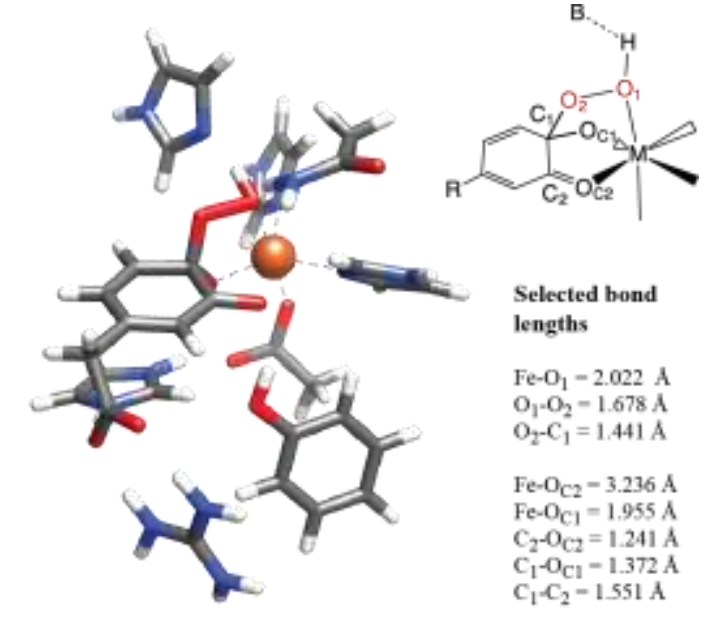

$\mathrm{TS2}_{\mathrm{C} 1}$

Figure S4 Calculated structures for $\mathrm{TS}_{\mathrm{C} 1}$ and $\mathrm{TS} 2_{\mathrm{C} 2}$. 

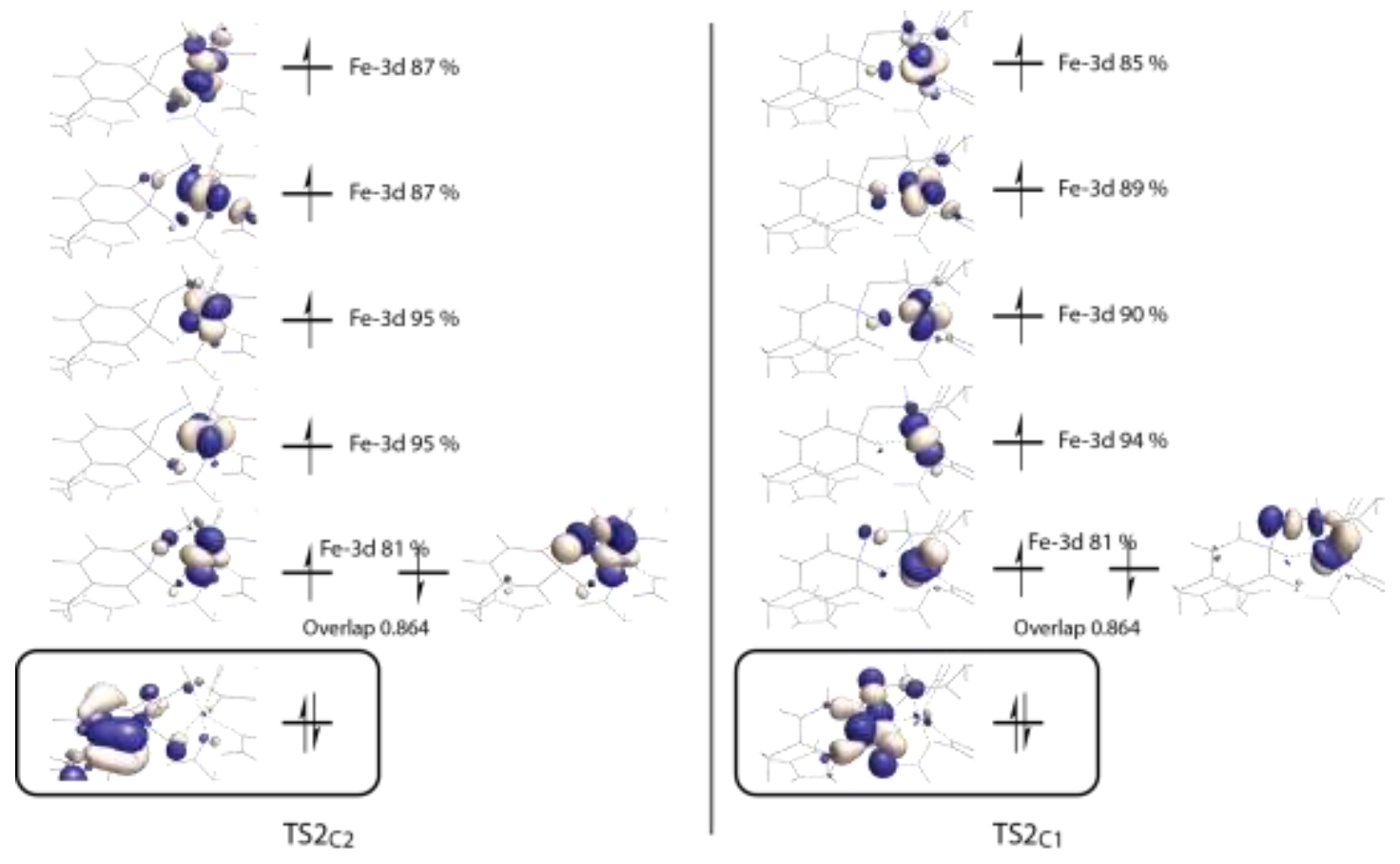

Figure S5 Electronic structure of TS2 $2_{1}$ and TS2 $2_{2}$ including the highest lying substrate based molecular orbital. The percentage of $d$ orbital character in each of the metal based orbitals is also included.

\section{The structure of the quasi- intermediate}
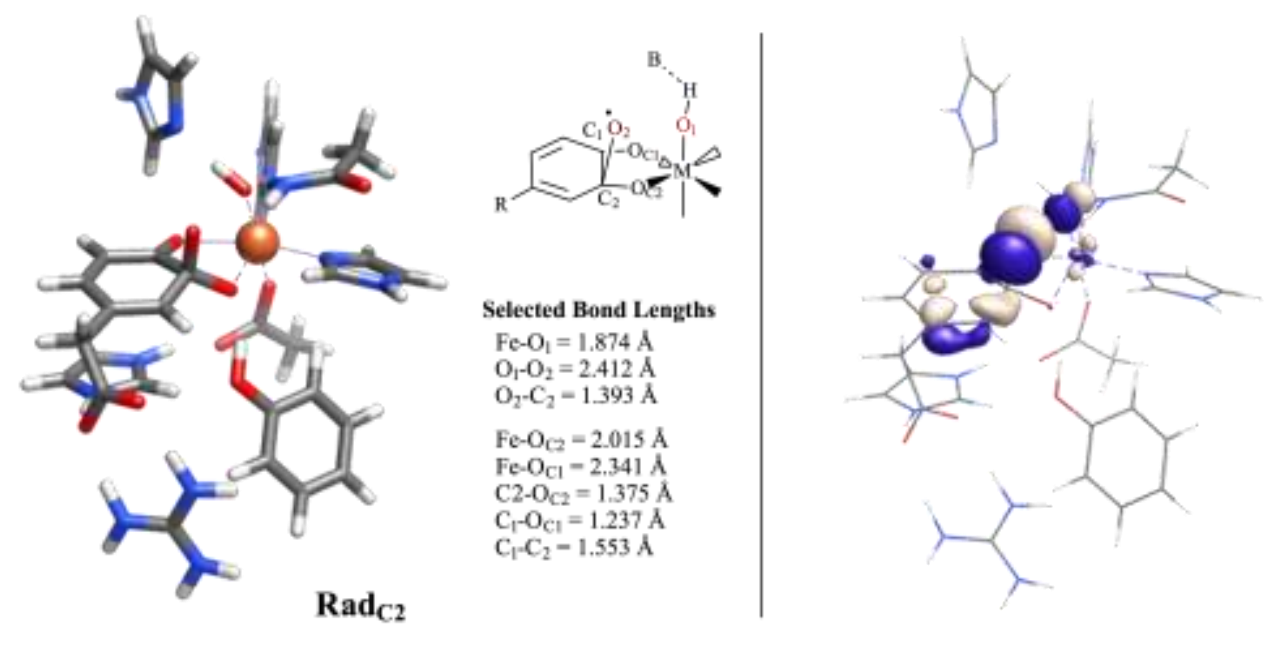

Figure S6 Optimized structure of the quasi-intermediate on the $\mathrm{C}_{2}$ pathway and the spin down UCO showing the radical character on the oxyl radical.

\section{Relationship between orbital energies and the barrier to intradiol cleavage}




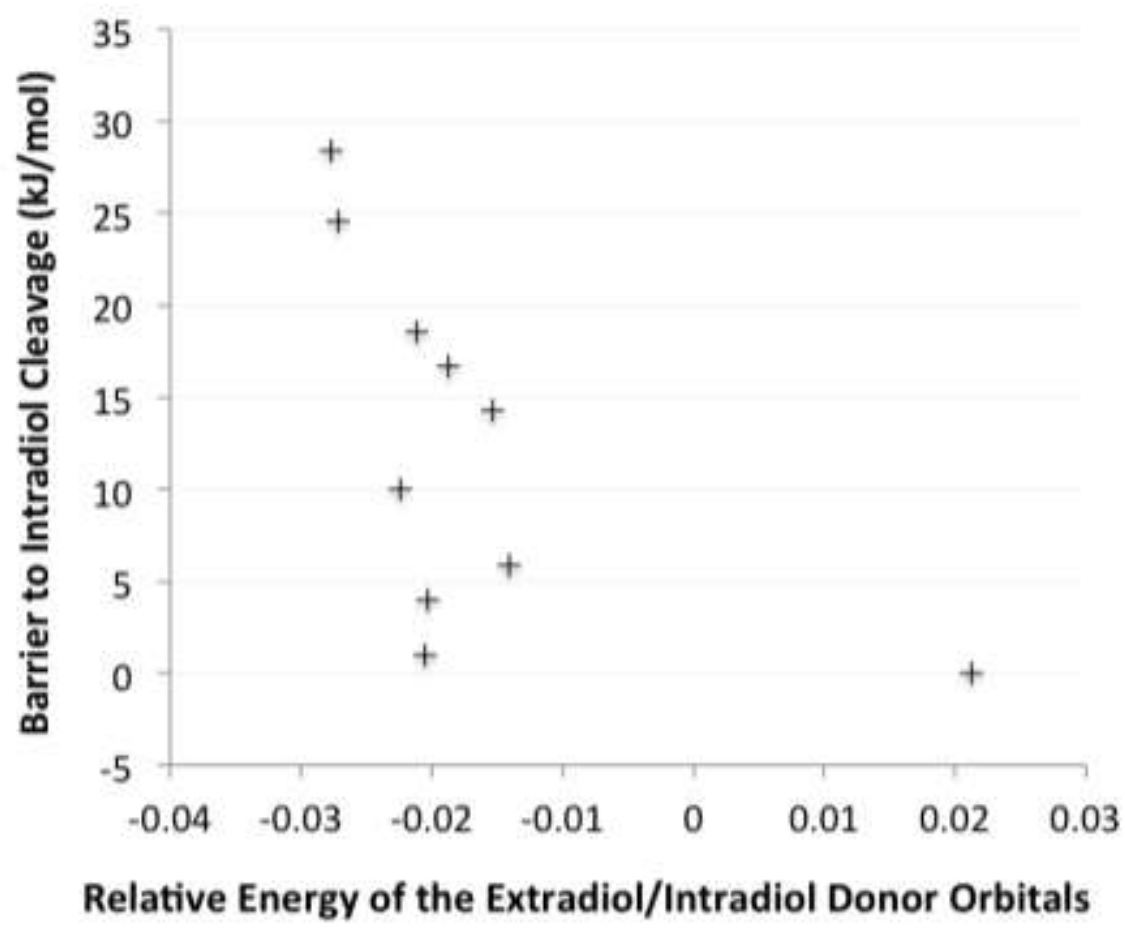

Figure S7 Plot of the relationship between the barrier to the intradiol cleavage and the relative energy of the extradiol and intradiol electron donating orbitals (EDOs).

The reason for the reversed preference starting from $\mathrm{Br}_{\mathrm{C} 1}$ can be traced back to the different electronic strucute of $\mathrm{TS} 2_{\mathrm{C} 1}$ and $\mathrm{TS} 2_{\mathrm{C} 2}$. The breaking of the 0-0 bond occurs via TS2, leading to the oxyl quasi intermediate. The calculated geometry and electronic structure of TS2 $2_{1}$ is shown in Figure S8. Analysis of the electronic structure shows that for $\mathrm{TS} 2 \mathrm{C} 1$ and $\mathrm{TS} 2 \mathrm{C} 2$ are similar with respect to the metal and oxygen interaction. For both transition states the metal is best described as having a Fe $/$ II $/ \mathrm{Fe}^{\mathrm{III}}$ center with some radical character on the peroxo species. This indicates partial electron transfer from the metal to the $\mathrm{O}_{2} \sigma^{*}$ orbital. Despite these similarities, the HOMO of the substrate is distinctly different for $\mathrm{TS}_{2} \mathrm{C}_{2}$. For $\mathrm{TS}_{\mathrm{C} 2}$ the substrate HOMO is predominantly the extradiol EDO in character (Figure S8). Therefore one may expect that the extradiol pathway is facile, consistent with the theoretical results. While, the substrate HOMO for TS2 $2_{1}$ is mainly the intradiol EDO. Thus, intradiol cleavage starting from $\mathrm{TS}_{\mathrm{C} 1}$ may traverse a lower barrier than that from $\mathrm{TS} 2_{\mathrm{C} 2}$. 

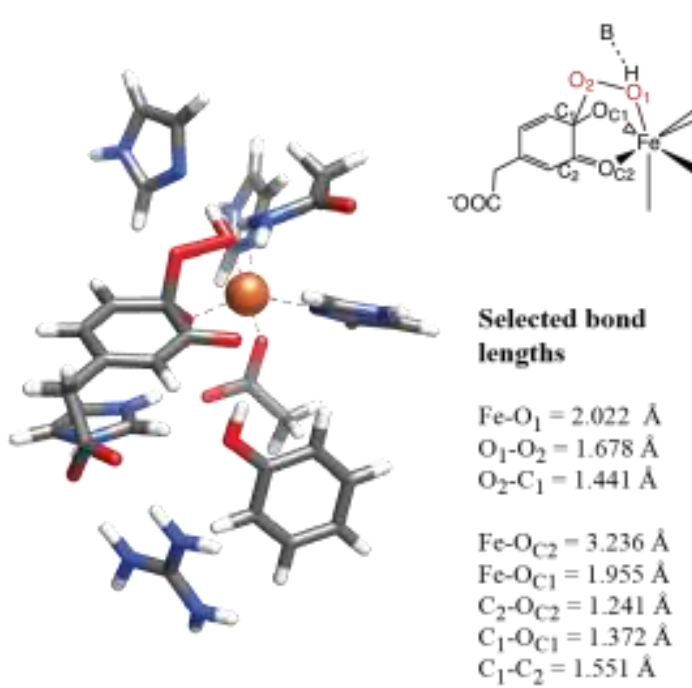

Optimised structure $\mathrm{TS}_{\mathrm{C} 1}$

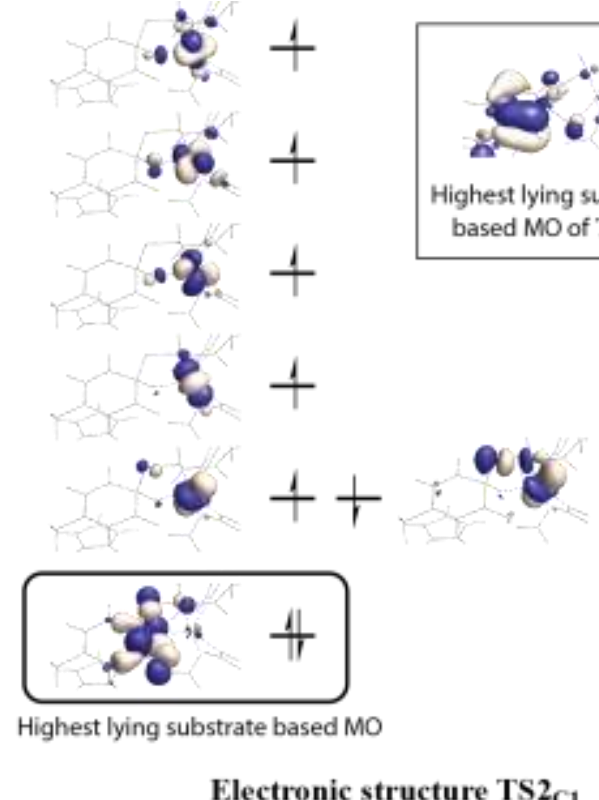

Electronic structure $\mathbf{T S} \mathbf{2}_{\mathrm{C} 1}$

Figure S8 Optimized structure and electronic structure of TS2 $2_{\mathrm{C} 1}$. The highest lying substrate based molecular orbital for $\mathrm{TS}_{\mathrm{C} 2}$ is included for comparison. 


\section{Cartesion coordinates of the calculated structures of key species}

TS1 1

\begin{tabular}{|c|c|c|c|}
\hline C & -0.589222 & 3.577465 & -4.044627 \\
\hline $\mathrm{N}$ & -0.961140 & 4.284644 & -2.916188 \\
\hline $\mathrm{C}$ & -0.700246 & 2.253171 & -3.686229 \\
\hline $\mathrm{C}$ & -1.279188 & 3.402639 & -1.941899 \\
\hline $\mathrm{N}$ & -1.126596 & 2.158660 & -2.372355 \\
\hline $\mathrm{C}$ & -0.700544 & -2.073298 & -4.596429 \\
\hline $\mathrm{C}$ & -0.255258 & -3.298333 & -3.815814 \\
\hline 0 & -0.780830 & -4.400537 & -3.995720 \\
\hline $\mathrm{N}$ & 0.759089 & -3.106540 & -2.938597 \\
\hline $\mathrm{C}$ & 3.570824 & 3.219002 & -3.624825 \\
\hline $\mathrm{N}$ & 3.031857 & 3.757112 & -2.47 \\
\hline $\mathrm{C}$ & 3.032340 & 1.958132 & -3.724782 \\
\hline $\mathrm{C}$ & 2.207061 & 2.833417 & -1.921770 \\
\hline $\mathrm{N}$ & 2.188147 & 1.738574 & -2.6 \\
\hline $\mathrm{C}$ & -4.243200 & -2.007362 & -2.636357 \\
\hline $\mathrm{N}$ & -3.290201 & -2.920850 & -2.2 \\
\hline $\mathrm{C}$ & -3.709147 & -0.772765 & -2.345514 \\
\hline $\mathrm{C}$ & -2.239787 & -2.246141 & -1.722625 \\
\hline $\mathrm{N}$ & -2.457554 & -0.939873 & -1.781048 \\
\hline $\mathrm{N}$ & -2.7 & -4.738258 & 6.7 \\
\hline $\mathrm{C}$ & -1.494822 & -4.522335 & 6.229691 \\
\hline $\mathrm{N}$ & -1.234989 & -4.946631 & 4.993648 \\
\hline $\mathrm{N}$ & 410 & -3.869397 & 6.909660 \\
\hline $\mathrm{C}$ & 1.069579 & 1.088210 & 5.280102 \\
\hline $\mathrm{N}$ & 0.621236 & -0.188830 & 5.016252 \\
\hline $\mathrm{C}$ & 0.343190 & 1.931463 & 4.477833 \\
\hline $\mathrm{C}$ & -0.341303 & -0.133364 & 4.092070 \\
\hline $\mathrm{N}$ & -0.520859 & 1.140842 & 3.747501 \\
\hline $\mathrm{C}$ & -5.957715 & -2.71 & 2.9 \\
\hline $\mathrm{C}$ & -5.407232 & -2.430325 & 1.696146 \\
\hline $\mathrm{C}$ & -5.120950 & -2.851920 & 4.065363 \\
\hline $\mathrm{C}$ & -4.023567 & -2.308875 & 1.542140 \\
\hline $\mathrm{C}$ & -3.737516 & -2.731064 & 3.923824 \\
\hline $\mathrm{C}$ & -3.189503 & -2.478379 & 2.657932 \\
\hline 0 & -1.831858 & -2.411166 & 2.562914 \\
\hline $\mathrm{C}$ & -4.790071 & 1.315231 & 1.402413 \\
\hline $\mathrm{C}$ & -3.285978 & 1.477550 & 1.204368 \\
\hline 0 & -2.824995 & 1.382882 & 0.019916 \\
\hline 0 & -2.603353 & 1.708454 & 2.233815 \\
\hline $\mathrm{Fe}$ & -1.184512 & 0.543985 & -0.902872 \\
\hline $\mathrm{C}$ & 2.292191 & -1.691994 & 2.457926 \\
\hline $\mathrm{C}$ & 1.114740 & -1.996126 & 1.833035 \\
\hline $\mathrm{C}$ & 0.482163 & -1.031093 & 0.971848 \\
\hline $\mathrm{C}$ & 1.211167 & 0.227258 & 0.593066 \\
\hline $\mathrm{C}$ & 2.485470 & 0.477102 & 1.293325 \\
\hline $\mathrm{C}$ & 2.981876 & -0.430607 & 2.166359 \\
\hline $\mathrm{C}$ & 2.898107 & -2.630403 & 3.483759 \\
\hline $\mathrm{C}$ & 1.822212 & -3.309838 & 4.354827 \\
\hline 0 & 1.315772 & -4.379404 & 3.953728 \\
\hline 0 & 1.482014 & -2.701086 & 5.429337 \\
\hline 0 & -0.692197 & -1.155980 & 0.541280 \\
\hline 0 & 0.493815 & 1.200130 & 0.154033 \\
\hline
\end{tabular}

$\begin{array}{rrrr}\mathrm{H} & -0.485380 & 1.367406 & -4.272534 \\ \mathrm{H} & -1.012561 & 5.295249 & -2.835631 \\ \mathrm{H} & -1.616071 & 3.681236 & -0.949279 \\ \mathrm{H} & -0.281609 & -1.139272 & -4.204069 \\ \mathrm{H} & -1.796151 & -2.012101 & -4.580944 \\ \mathrm{H} & 1.053876 & -3.893429 & -2.366559 \\ \mathrm{H} & 1.022835 & -2.166725 & -2.636297 \\ \mathrm{H} & 3.198729 & 1.202814 & -4.485872\end{array}$

H $\quad 3.232932 \quad 4.677001 \quad-2.095492$

$\mathrm{H} \quad 1.645380 \quad 2.972858-1.004349$

H $\quad-4.131771 \quad 0.215723 \quad-2.480080$

H $\quad-3.336430 \quad-3.929101-2.346974$

H $-1.353438 \quad-2.715246 \quad-1.318033$

$\begin{array}{llll}\mathrm{H} & -2.893681 & -4.437081 & 7.734765\end{array}$

$\mathrm{H} \quad-0.300341 \quad-4.764244 \quad 4.567850$

H $\quad-1.992599 \quad-5.233500 \quad 4.382696$

$\begin{array}{llll}\mathrm{H} & -0.709404 & -3.600467 & 7.875017\end{array}$

$\mathrm{H} \quad 0.277322 \quad-3.475282 \quad 6.408404$

$\mathrm{H} \quad 0.364886 \quad 3.008579 \quad 4.368036$

H $\quad 1.029817 \quad-1.097883 \quad 5.349999$

$\mathrm{H} \quad-1.253764 \quad 1.447566 \quad 3.046766$

$\mathrm{H} \quad-0.881462 \quad-0.984018 \quad 3.682749$

$\begin{array}{llll}\mathrm{H} & -6.056369 & -2.300260 & 0.829459\end{array}$

H $\quad-5.547872 \quad-3.051623 \quad 5.049195$

$\mathrm{H} \quad-3.589070 \quad-2.076986 \quad 0.569670$

$\begin{array}{llll}\mathrm{H} & -3.071208 & -2.815646 & 4.779799\end{array}$

$\mathrm{H} \quad-1.555584 \quad-2.083669 \quad 1.667126$

$\begin{array}{llll}\mathrm{H} & -4.962093 & 0.472101 & 2.085915\end{array}$

$\begin{array}{llll}\mathrm{H} & -5.196925 & 2.216722 & 1.882095\end{array}$

H $\quad 0.585928 \quad-2.921357 \quad 2.051472$

$\mathrm{H} \quad 2.993236 \quad 1.417210 \quad 1.081440$

H $\quad 3.919970 \quad-0.231940 \quad 2.685544$

$\begin{array}{llll}\mathrm{H} & 3.596832 & -2.080658 & 4.125971\end{array}$

$\begin{array}{llll}\mathrm{H} & 3.468176 & -3.411748 & 2.958302\end{array}$

H $1.348849 \quad 0.489109-2.260658$

H $4.275095 \quad 3.761153 \quad-4.240894$

$\mathrm{H} \quad-0.270200 \quad 4.082700 \quad-4.958800$

$\mathrm{H} \quad-0.389400 \quad-2.199100 \quad-5.642800$

$\mathrm{H} \quad-5.190900 \quad-2.322200 \quad-3.064101$

$\begin{array}{llll}\mathrm{H} & -5.325797 & 1.123603 & 0.465899\end{array}$

$\mathrm{H} \quad-3.352102 \quad-5.417098 \quad 6.393199$

$\begin{array}{llll}\mathrm{H} & 1.839001 & 1.274602 & 6.019600\end{array}$

$\mathrm{H} \quad-7.029002 \quad-2.820794 \quad 3.066700$

$\begin{array}{llll}0 & 0.875285 & -0.361284 & -1.877299\end{array}$

$\begin{array}{llll}0 & 1.807742 & -0.735228 & -0.867415\end{array}$

\section{Brc1}

$\begin{array}{llll}\mathrm{C} & -0.595147 & 3.568396 & -4.050859 \\ \mathrm{~N} & -0.944175 & 4.263426 & -2.907476 \\ \mathrm{C} & -0.727628 & 2.240589 & -3.712598 \\ \mathrm{C} & -1.270832 & 3.371286 & -1.944970 \\ \mathrm{~N} & -1.144283 & 2.131544 & -2.396329\end{array}$




\begin{tabular}{|c|c|c|c|}
\hline & 33 & 7 & \\
\hline & -0.260842 & & \\
\hline & -0.809497 & & \\
\hline & 637 & -3 & \\
\hline & 3.561382 & & -36753 \\
\hline & 3.017218 & 3.628171 & -2.4901 \\
\hline & 3.018 & & \\
\hline & 2.186247 & & \\
\hline & 166581 & 910 & \\
\hline & -4.237590 & $-2 .($ & \\
\hline & 970 & 642 & \\
\hline & 532 & & \\
\hline & -2.228207 & 508 & \\
\hline & -2.435901 & 859 & \\
\hline & -2.7 & & \\
\hline & -1.4 & & \\
\hline & -1.2 & & \\
\hline & -0.5 & & \\
\hline & & & \\
\hline & & & \\
\hline & 0.37 & & \\
\hline & & & \\
\hline & -0.4 & & \\
\hline & & & \\
\hline & -5 & & \\
\hline & -5.1 & -2 & \\
\hline & & & \\
\hline & -3.7 & & \\
\hline & -3.1 & & \\
\hline & -1.8 & & \\
\hline & -4.7 & & \\
\hline & -3.27 & & \\
\hline & -2.8 & & \\
\hline & -2. & & \\
\hline & 245 & & \\
\hline & & & \\
\hline & & & \\
\hline & & & \\
\hline & 68 & & \\
\hline & 2.5 & & \\
\hline & & -0.4 & \\
\hline & & & \\
\hline & & & \\
\hline & 25 & -4 & \\
\hline & & & \\
\hline & -0.6 & & 36 \\
\hline & & & \\
\hline & & & \\
\hline & -0.9 & & \\
\hline & -1.594975 & & -0. \\
\hline & & & \\
\hline & & & \\
\hline & & & \\
\hline & & & \\
\hline & 3.187037 & & -4 \\
\hline & & & \\
\hline & & & \\
\hline & & & \\
\hline & -3.3 & -3.94 & \\
\hline
\end{tabular}

$\begin{array}{cccc}\mathrm{H} & -1.342221 & -2.765429 & -1.358497 \\ \mathrm{H} & -2.918157 & -4.487024 & 7.778731 \\ \mathrm{H} & -0.281661 & -4.715399 & 4.639367 \\ \mathrm{H} & -1.987983 & -5.091808 & 4.396182 \\ \mathrm{H} & -0.706373 & -3.723904 & 8.000631 \\ \mathrm{H} & 0.277526 & -3.498948 & 6.543133 \\ \mathrm{H} & 0.420554 & 2.988344 & 4.294935 \\ \mathrm{H} & 0.992367 & -1.093910 & 5.418896 \\ \mathrm{H} & -1.221551 & 1.418629 & 3.007568 \\ \mathrm{H} & -0.891439 & -0.998168 & 3.709056 \\ \mathrm{H} & -6.007208 & -2.460218 & 0.819048 \\ \mathrm{H} & -5.583517 & -2.940929 & 5.087110 \\ \mathrm{H} & -3.533411 & -2.278656 & 0.592109 \\ \mathrm{H} & -3.101103 & -2.747735 & 4.851262 \\ \mathrm{H} & -1.519031 & -2.233342 & 1.725108 \\ \mathrm{H} & -4.931474 & 0.318976 & 2.005441 \\ \mathrm{H} & -5.187050 & 2.072362 & 1.983241 \\ \mathrm{H} & 0.620785 & -3.005604 & 2.167961 \\ \mathrm{H} & 3.007517 & 1.284201 & 0.946766 \\ \mathrm{H} & 3.845370 & -0.176455 & 2.763857 \\ \mathrm{H} & 3.529805 & -1.946099 & 4.279544 \\ \mathrm{H} & 3.536837 & -3.328462 & 3.168566 \\ \mathrm{H} & 1.369892 & 0.349070 & -2.405729 \\ \mathrm{H} & 4.275095 & 3.761153 & -4.240894 \\ \mathrm{H} & -0.270200 & 4.082700 & -4.958800 \\ \mathrm{H} & -0.389400 & -2.199100 & -5.642800 \\ \mathrm{H} & -5.190900 & -2.322200 & -3.064101 \\ \mathrm{H} & -5.325797 & 1.123604 & 0.465900 \\ \mathrm{H} & -3.352103 & -5.417098 & 6.393200 \\ \mathrm{H} & 1.839001 & 1.274601 & 6.019602 \\ \mathrm{H} & -7.029002 & -2.820794 & 3.066700 \\ \mathrm{O} & 0.946089 & -0.480715 & -1.934368 \\ \mathrm{O} & 1.906497 & -0.619759 & -0.858891\end{array}$

\section{TS2 1}

$\begin{array}{lccc}\mathrm{C} & -0.588612 & 3.579340 & -4.043531 \\ \mathrm{~N} & -0.982358 & 4.281590 & -2.918606 \\ \mathrm{C} & -0.669320 & 2.255342 & -3.682645 \\ \mathrm{C} & -1.284817 & 3.398570 & -1.941801 \\ \mathrm{~N} & -1.097921 & 2.156754 & -2.369236 \\ \mathrm{C} & 0.075704 & -1.904351 & -4.695790 \\ \mathrm{C} & 0.412606 & -3.154375 & -3.896704 \\ \mathrm{O} & 0.032155 & -4.275487 & -4.241374 \\ \mathrm{~N} & 1.151544 & -2.947626 & -2.777128 \\ \mathrm{C} & 3.584341 & 3.187781 & -3.634435 \\ \mathrm{~N} & 3.203745 & 3.596260 & -2.372663 \\ \mathrm{C} & 2.922621 & 2.000620 & -3.848174 \\ \mathrm{C} & 2.350091 & 2.670435 & -1.870791 \\ \mathrm{~N} & 2.160685 & 1.697068 & -2.741803 \\ \mathrm{C} & -4.228112 & -2.040039 & -2.647195 \\ \mathrm{~N} & -3.192214 & -2.947665 & -2.533325 \\ \mathrm{C} & -3.733805 & -0.859839 & -2.145789 \\ \mathrm{C} & -2.129954 & -2.323926 & -1.985830 \\ \mathrm{~N} & -2.421302 & -1.052369 & -1.742833 \\ \mathrm{~N} & -2.843417 & -4.629761 & 6.778415 \\ \mathrm{C} & -1.623225 & -4.311509 & 6.297240 \\ \mathrm{~N} & -1.010942 & -5.109470 & 5.428761\end{array}$ 


\begin{tabular}{|c|c|c|c|}
\hline & 51 & 16 & \\
\hline & 1.100847 & 1.127124 & \\
\hline & 0.658461 & -0.135346 & \\
\hline & & 2278 & \\
\hline & & .045501 & \\
\hline & -0.397656 & 1343 & \\
\hline & 582 & & \\
\hline & -5.348530 & 013 & \\
\hline & -5.1 & 156 & \\
\hline & -3.957018 & & \\
\hline & -3.7 & & \\
\hline & & & \\
\hline & -1.79 & 566 & 91 \\
\hline$C$ & -4.64 & & 6251 \\
\hline & -3.2 & & \\
\hline & & & \\
\hline & -2.5 & & \\
\hline e & -1.1 & & J. \\
\hline & 2.34 & & \\
\hline & & & \\
\hline & & 04 & \\
\hline & & & \\
\hline & & & \\
\hline & & & \\
\hline & 3.0 & & \\
\hline & & & \\
\hline & & & \\
\hline & & & \\
\hline & & & \\
\hline & -0. & & \\
\hline & & & 165 \\
\hline & & & \\
\hline & & & \\
\hline & & 28 & 254 \\
\hline & & & \\
\hline & & & -2 \\
\hline & & & \\
\hline & & & \\
\hline & 3.5 & & \\
\hline & & & \\
\hline & & & 42 \\
\hline & & & \\
\hline & & -2 & 899 \\
\hline & & & \\
\hline & & & \\
\hline & & & \\
\hline & & & \\
\hline & -0 . & 52 & \\
\hline & & & \\
\hline & & & 335 \\
\hline & & & \\
\hline & & & \\
\hline & & & \\
\hline & 944 & -2.401397 & 3456 \\
\hline & & & \\
\hline & & & \\
\hline & & & \\
\hline & -4.596 & -0.179754 & 1.42109 \\
\hline & -5.056859 & 1.333164 & 2.2318 \\
\hline
\end{tabular}

$\begin{array}{cccc}\mathrm{H} & 1.004215 & -3.577867 & 2.213610 \\ \mathrm{H} & 2.516311 & 1.130725 & 1.201400 \\ \mathrm{H} & 3.562752 & -0.179089 & 3.024411 \\ \mathrm{H} & 3.657802 & -2.058203 & 4.423472 \\ \mathrm{H} & 3.630126 & -3.542162 & 3.441987 \\ \mathrm{H} & 1.161060 & 0.543017 & -2.328016 \\ \mathrm{H} & 4.275095 & 3.761153 & -4.240894 \\ \mathrm{H} & -0.270200 & 4.082700 & -4.958800 \\ \mathrm{H} & -0.389400 & -2.199100 & -5.642800 \\ \mathrm{H} & -5.190900 & -2.322200 & -3.064101 \\ \mathrm{H} & -5.325797 & 1.123604 & 0.465900 \\ \mathrm{H} & -3.352103 & -5.417098 & 6.393200 \\ \mathrm{H} & 1.839001 & 1.274601 & 6.019602 \\ \mathrm{H} & -7.029002 & -2.820794 & 3.066700 \\ \mathrm{O} & 0.548267 & -0.179521 & -1.889993 \\ \mathrm{O} & 1.723994 & -0.482033 & -0.731582\end{array}$

quasi-intermediate $\mathrm{C}_{2}$

$\begin{array}{lccc}\mathrm{C} & -0.665378 & 3.580537 & -4.073469 \\ \mathrm{~N} & -1.445132 & 4.235860 & -3.135385 \\ \mathrm{C} & -0.540590 & 2.297015 & -3.599200 \\ \mathrm{C} & -1.764170 & 3.362291 & -2.154461 \\ \mathrm{~N} & -1.227577 & 2.175631 & -2.401759 \\ \mathrm{C} & -0.797719 & -2.108912 & -4.626219 \\ \mathrm{C} & -0.170438 & -3.197589 & -3.770661 \\ \mathrm{O} & -0.431755 & -4.391967 & -3.962731 \\ \mathrm{~N} & 0.694564 & -2.774591 & -2.825299 \\ \mathrm{C} & 3.634943 & 3.234051 & -3.544010 \\ \mathrm{~N} & 3.056327 & 3.863254 & -2.461403 \\ \mathrm{C} & 3.215720 & 1.925296 & -3.467354 \\ \mathrm{C} & 2.325591 & 2.940950 & -1.782826 \\ \mathrm{~N} & 2.404091 & 1.758975 & -2.364936 \\ \mathrm{C} & -4.252478 & -2.025799 & -2.614003 \\ \mathrm{~N} & -3.350202 & -2.950299 & -2.126278 \\ \mathrm{C} & -3.686507 & -0.797394 & -2.378486 \\ \mathrm{C} & -2.291407 & -2.292505 & -1.619531 \\ \mathrm{~N} & -2.464032 & -0.985024 & -1.763603 \\ \mathrm{~N} & -2.772377 & -4.693693 & 6.802557 \\ \mathrm{C} & -1.532970 & -4.468500 & 6.319815 \\ \mathrm{~N} & -1.176164 & -4.968216 & 5.137941 \\ \mathrm{~N} & -0.664638 & -3.734056 & 7.014974 \\ \mathrm{C} & 1.034827 & 1.139039 & 5.303757 \\ \mathrm{~N} & 0.515206 & -0.114248 & 5.063582 \\ \mathrm{C} & 0.349056 & 2.009358 & 4.492772 \\ \mathrm{C} & -0.439262 & -0.028253 & 4.136735 \\ \mathrm{~N} & -0.556165 & 1.250585 & 3.775059 \\ \mathrm{C} & -5.965799 & -2.638659 & 2.909528 \\ \mathrm{C} & -5.496349 & -2.065701 & 1.719174 \\ \mathrm{C} & -5.046738 & -2.979034 & 3.911929 \\ \mathrm{C} & -4.126970 & -1.847103 & 1.516029 \\ \mathrm{C} & -3.680517 & -2.765702 & 3.720672 \\ \mathrm{C} & -3.209481 & -2.213271 & 2.516694 \\ \mathrm{O} & -1.865428 & -2.066271 & 2.392428 \\ \mathrm{C} & -4.730638 & 1.922710 & 0.940632 \\ \mathrm{C} & -3.233372 & 1.612504 & 1.011440 \\ \mathrm{O} & -2.664148 & 1.347736 & -0.112383 \\ \mathrm{O} & -2.649519 & 1.688471 & 2.109299\end{array}$




\begin{tabular}{|c|c|c|c|}
\hline & & & \\
\hline & & -1.450729 & \\
\hline & 173 & & \\
\hline & 519 & & \\
\hline & 1.128790 & 6433 & \\
\hline & & & \\
\hline & 781 & & \\
\hline & 672 & & \\
\hline & 725912 & -3.160774 & 0771 \\
\hline & & & \\
\hline & & & \\
\hline & 995 & & \\
\hline & 723 & & \\
\hline & & & \\
\hline & & & \\
\hline & & & \\
\hline & & -1. & \\
\hline & 071 & -2.2 & \\
\hline & & & \\
\hline & & & \\
\hline & & & \\
\hline & & & \\
\hline & & & \\
\hline & & & \\
\hline & & & \\
\hline & & & \\
\hline & $-3 .($ & & \\
\hline & & & \\
\hline & & & \\
\hline & & & \\
\hline & & & \\
\hline & & & \\
\hline & & & \\
\hline & & & \\
\hline & & & \\
\hline & & & \\
\hline & & & \\
\hline & & & \\
\hline & -2. & & \\
\hline & & & \\
\hline & & & \\
\hline & & & \\
\hline & & & \\
\hline & & & \\
\hline & & & \\
\hline & & & \\
\hline & & & \\
\hline & & & \\
\hline & & & \\
\hline & & & \\
\hline & & & \\
\hline & & & \\
\hline & & & \\
\hline & & -5.417098 & 3199 \\
\hline & & & 600 \\
\hline & & & \\
\hline & & & \\
\hline & & -1.363711 & -0.317 \\
\hline
\end{tabular}

\section{quaasi-intermediate $C_{1}$}

\begin{tabular}{lccc}
$\mathrm{C}$ & -0.553414 & 3.610077 & -4.023133 \\
$\mathrm{~N}$ & -0.867900 & 4.324083 & -2.881355 \\
$\mathrm{C}$ & -0.623285 & 2.290676 & -3.657477 \\
$\mathrm{C}$ & -1.121575 & 3.450067 & -1.884481 \\
$\mathrm{~N}$ & -0.976454 & 2.208562 & -2.325802 \\
$\mathrm{C}$ & 0.676611 & -2.174677 & -5.368575 \\
$\mathrm{C}$ & 0.971789 & -3.283287 & -4.369556 \\
$\mathrm{O}$ & 1.001777 & -4.469894 & -4.713155 \\
$\mathrm{~N}$ & 1.194456 & -2.881165 & -3.095923 \\
$\mathrm{C}$ & 3.840042 & 2.895897 & -3.761827 \\
$\mathrm{~N}$ & 4.413306 & 2.295940 & -2.659870 \\
$\mathrm{C}$ & 2.696410 & 2.175705 & -4.011105 \\
$\mathrm{C}$ & 3.613833 & 1.262108 & -2.286854 \\
$\mathrm{~N}$ & 2.563787 & 1.164959 & -3.081061 \\
$\mathrm{C}$ & -4.213857 & -2.033883 & -2.698949 \\
$\mathrm{~N}$ & -3.107063 & -2.830889 & -2.912353 \\
$\mathrm{C}$ & -3.746255 & -0.923480 & -2.039519 \\
$\mathrm{C}$ & -2.024945 & -2.207473 & -2.405976 \\
$\mathrm{~N}$ & -2.378354 & -1.047782 & -1.870517 \\
$\mathrm{~N}$ & -2.771266 & -4.750864 & 6.887028 \\
$\mathrm{C}$ & -1.525304 & -4.495835 & 6.437010 \\
$\mathrm{~N}$ & -0.981604 & -5.273836 & 5.504557 \\
$\mathrm{~N}$ & -0.826096 & -3.489364 & 6.959470 \\
$\mathrm{C}$ & 1.096868 & 1.102967 & 5.236448 \\
$\mathrm{~N}$ & 0.625824 & -0.167183 & 4.968089 \\
$\mathrm{C}$ & 0.432757 & 1.947850 & 4.378817 \\
$\mathrm{C}$ & -0.282902 & -0.108592 & 3.991765 \\
$\mathrm{~N}$ & -0.416749 & 1.164576 & 3.616973 \\
$\mathrm{C}$ & -5.946029 & -2.744832 & 2.985573 \\
$\mathrm{C}$ & -5.336978 & -2.645903 & 1.727833 \\
$\mathrm{C}$ & -5.151189 & -2.751091 & 4.139779 \\
$\mathrm{C}$ & -3.945227 & -2.566860 & 1.614207 \\
$\mathrm{C}$ & -3.758768 & -2.677557 & 4.039362 \\
$\mathrm{C}$ & -3.157534 & -2.595524 & 2.775313 \\
$\mathrm{O}$ & -1.790801 & -2.546192 & 2.724682 \\
$\mathrm{C}$ & -4.653113 & 1.969559 & 0.718419 \\
$\mathrm{C}$ & -3.164442 & 1.588624 & 0.828649 \\
$\mathrm{O}$ & -2.609299 & 1.220999 & -0.270829 \\
$\mathrm{O}$ & -2.581674 & 1.705479 & 1.927338 \\
$\mathrm{H}$ & -1.406695 & 3.732368 & -0.877590 \\
$\mathrm{~F}$ & -0.973979 & 0.410543 & -1.210181 \\
$\mathrm{H}$ & 0.910512 & -1.174913 & -6.281312 \\
$\mathrm{C}$ & 2.299648 & -1.941209 & 2.686146 \\
$\mathrm{C}$ & 1.282093 & -2.441103 & 1.919128 \\
$\mathrm{C}$ & 0.601707 & -1.630883 & 0.926113 \\
$\mathrm{C}$ & 1.248844 & -0.275188 & 0.491839 \\
$\mathrm{C}$ & 2.266154 & 0.228373 & 1.525691 \\
$\mathrm{C}$ & 2.750006 & -0.562781 & 2.507937 \\
$\mathrm{C}$ & 2.947167 & -2.790082 & 3.761717 \\
$\mathrm{C}$ & 1.892410 & -3.405869 & 4.705379 \\
$\mathrm{O}$ & 1.526264 & -4.581835 & 4.505916 \\
$\mathrm{O}$ & 1.433681 & -2.642167 & 5.625167 \\
$\mathrm{O}$ & -0.456537 & -1.965183 & 0.386776 \\
$\mathrm{H}$ & -0.422825 & 1.39206 & 0.202960 \\
\hline
\end{tabular}




$\begin{array}{cccc}\mathrm{H} & 1.374498 & -3.595570 & -2.395572 \\ \mathrm{H} & 0.998565 & -1.912724 & -2.787012 \\ \mathrm{H} & 1.963617 & 2.333202 & -4.794024 \\ \mathrm{H} & 5.276071 & 2.577494 & -2.205595 \\ \mathrm{H} & 3.827342 & 0.619978 & -1.440284 \\ \mathrm{H} & -4.267996 & -0.048534 & -1.673389 \\ \mathrm{H} & -3.103133 & -3.733789 & -3.377228 \\ \mathrm{H} & -1.018532 & -2.601269 & -2.448242 \\ \mathrm{H} & -3.257562 & -4.067936 & 7.457979 \\ \mathrm{H} & -0.028754 & -5.069808 & 5.130159 \\ \mathrm{H} & -1.539040 & -5.977487 & 5.033047 \\ \mathrm{H} & -1.227386 & -2.910489 & 7.688610 \\ \mathrm{H} & 0.085699 & -3.203687 & 6.535673 \\ \mathrm{H} & 0.490196 & 3.021550 & 4.248125 \\ \mathrm{H} & 0.995775 & -1.078232 & 5.350666 \\ \mathrm{H} & -1.094929 & 1.464500 & 2.875019 \\ \mathrm{H} & -0.822064 & -0.955597 & 3.571318 \\ \mathrm{H} & -5.946854 & -2.630375 & 0.823886 \\ \mathrm{H} & -5.619849 & -2.817157 & 5.123029 \\ \mathrm{H} & -3.468815 & -2.476607 & 0.635950 \\ \mathrm{H} & -3.122678 & -2.683435 & 4.923904 \\ \mathrm{H} & -1.485625 & -2.514745 & 1.788140 \\ \mathrm{H} & -5.001122 & 2.413637 & 1.658877 \\ \mathrm{H} & -4.769458 & 2.706052 & -0.089721 \\ \mathrm{H} & 0.874726 & -3.433484 & 2.113567 \\ \mathrm{H} & 2.616045 & 1.244118 & 1.351144 \\ \mathrm{H} & 3.480165 & -0.164966 & 3.213102 \\ \mathrm{H} & 3.650192 & -2.190140 & 4.351406 \\ \mathrm{H} & 3.507493 & -3.605771 & 3.284223 \\ \mathrm{H} & 1.093874 & 0.204222 & -2.596685 \\ \mathrm{H} & 4.275095 & 3.761153 & -4.240894 \\ \mathrm{H} & -0.270200 & 4.082700 & -4.958800 \\ \mathrm{H} & -0.389400 & -2.199100 & -5.642800 \\ \mathrm{H} & -5.190901 & -2.322199 & -3.064101 \\ \mathrm{H} & -5.325798 & 1.123605 & 0.465899 \\ \mathrm{H} & -3.352104 & -5.417096 & 6.393201 \\ \mathrm{H} & 1.839000 & 1.274604 & 6.019603 \\ \mathrm{H} & -7.029003 & -2.820792 & 3.066701 \\ \mathrm{O} & 0.256412 & -0.314489 & -2.433755 \\ \mathrm{O} & 2.140747 & -0.642065 & -0.490893\end{array}$

\section{TS3 2}

$\begin{array}{lccc}\mathrm{C} & -0.666583 & 3.606167 & -4.061205 \\ \mathrm{~N} & -1.278275 & 4.311239 & -3.038318 \\ \mathrm{C} & -0.666284 & 2.298878 & -3.643418 \\ \mathrm{C} & -1.623609 & 3.437333 & -2.063326 \\ \mathrm{~N} & -1.261454 & 2.207393 & -2.395916 \\ \mathrm{C} & -0.816818 & -2.158001 & -4.630185 \\ \mathrm{C} & -0.120367 & -3.202374 & -3.769611 \\ \mathrm{O} & -0.317827 & -4.411862 & -3.946305 \\ \mathrm{~N} & 0.725048 & -2.721839 & -2.834586 \\ \mathrm{C} & 3.598055 & 3.256639 & -3.565695 \\ \mathrm{~N} & 2.927783 & 3.928982 & -2.565785 \\ \mathrm{C} & 3.202785 & 1.944167 & -3.447770 \\ \mathrm{C} & 2.168642 & 3.025560 & -1.893027 \\ \mathrm{~N} & 2.315803 & 1.816461 & -2.400200 \\ \mathrm{C} & -4.250663 & -2.057357 & -2.605998\end{array}$

\begin{tabular}{cccc}
$\mathrm{N}$ & -3.366484 & -3.005398 & -2.132737 \\
$\mathrm{C}$ & -3.665095 & -0.843352 & -2.355224 \\
$\mathrm{C}$ & -2.294256 & -2.374355 & -1.619869 \\
$\mathrm{~N}$ & -2.446681 & -1.062905 & -1.746203 \\
$\mathrm{~N}$ & -2.820357 & -4.662643 & 6.809372 \\
$\mathrm{C}$ & -1.564669 & -4.427742 & 6.375580 \\
$\mathrm{~N}$ & -1.156486 & -4.947415 & 5.220388 \\
$\mathrm{~N}$ & -0.737078 & -3.663815 & 7.088207 \\
$\mathrm{C}$ & 1.026101 & 1.154012 & 5.309337 \\
$\mathrm{~N}$ & 0.489978 & -0.091711 & 5.066943 \\
$\mathrm{C}$ & 0.349002 & 2.035822 & 4.502500 \\
$\mathrm{C}$ & -0.462194 & 0.008172 & 4.140561 \\
$\mathrm{~N}$ & -0.565988 & 1.289788 & 3.782680 \\
$\mathrm{C}$ & -5.966317 & -2.634346 & 2.898948 \\
$\mathrm{C}$ & -5.509593 & -2.048540 & 1.709574 \\
$\mathrm{C}$ & -5.034194 & -2.982079 & 3.887250 \\
$\mathrm{C}$ & -4.142635 & -1.819265 & 1.495572 \\
$\mathrm{C}$ & -3.670636 & -2.759620 & 3.685182 \\
$\mathrm{C}$ & -3.215086 & -2.187216 & 2.484888 \\
$\mathrm{O}$ & -1.869766 & -2.022965 & 2.360934 \\
$\mathrm{C}$ & -4.687718 & 1.962498 & 0.803941 \\
$\mathrm{C}$ & -3.206919 & 1.599963 & 0.982515 \\
$\mathrm{O}$ & -2.581352 & 1.256459 & -0.086675 \\
$\mathrm{O}$ & -2.684281 & 1.710799 & 2.109124 \\
$\mathrm{Fe}$ & -1.089063 & 0.363829 & -1.091671 \\
$\mathrm{C}$ & 2.208031 & -1.489164 & 2.729677 \\
$\mathrm{C}$ & 1.206340 & -1.859497 & 1.894139 \\
$\mathrm{C}$ & 0.797521 & -1.107029 & 0.638202 \\
$\mathrm{C}$ & 1.297699 & 0.708817 & 0.943500 \\
$\mathrm{C}$ & 2.397544 & 0.863775 & 1.843666 \\
$\mathrm{C}$ & 2.796161 & -0.159823 & 2.653045 \\
$\mathrm{C}$ & 2.797496 & -2.457738 & 3.754221 \\
$\mathrm{C}$ & 1.723252 & -3.172986 & 4.578128 \\
$\mathrm{O}$ & 1.310269 & -4.287372 & 4.193797 \\
$\mathrm{O}$ & 1.283424 & -2.554038 & 5.613968 \\
$\mathrm{O}$ & -0.515908 & -0.930932 & 0.399602 \\
$\mathrm{O}$ & 0.666136 & 1.483327 & 0.280446 \\
$\mathrm{H}$ & -0.259613 & 1.419397 & -4.123673 \\
$\mathrm{H}$ & 0.110110 & -3.266193 & 6.619668 \\
$\mathrm{H}$ & 0.853773 & -1.022168 & 5.419557 \\
$\mathrm{H}$ & -1.267229 & 1.601747 & 3.068845 \\
$\mathrm{H}$ & -1.032905 & -0.811943 & 3.708536 \\
$\mathrm{H}$ & -2.121454 & 5.310819 & -3.022564 \\
$\mathrm{H}$ & -0.705102 & -1.117941 & -1.141851 \\
$\mathrm{H}$ & -1.812817 & -4.232217 \\
$\mathrm{H}$ & -1.882076 & -2.411507 & -4.710690 \\
$\mathrm{H}$ & 1.177717 & -3.382002 & -2.207978 \\
$\mathrm{H}$ & 0.712671 & -1.725228 & -2.554547 \\
$\mathrm{H}$ & 3.499131 & 1.089150 & -4.047717 \\
$\mathrm{H}$ & 2.999280 & 4.920307 & -2.361190 \\
$\mathrm{H}$ & 1.524562 & 3.290834 & -1.062029 \\
$\mathrm{H}$ & -4.020787 & 0.160216 & -2.553975 \\
$\mathrm{H}$ & -3.488701 & -4.012935 & -2.174817 \\
$\mathrm{H}$ & -1.439119 & -2.862845 & -1.172569 \\
$\mathrm{H}$ & -3.106269 & -4.370035 & 7.738090 \\
\hline
\end{tabular}




$\begin{array}{cccc}\mathrm{H} & -6.221413 & -1.762340 & 0.933955 \\ \mathrm{H} & -5.371701 & -3.428102 & 4.824417 \\ \mathrm{H} & -3.795376 & -1.354478 & 0.572392 \\ \mathrm{H} & -2.936659 & -3.019340 & 4.445669 \\ \mathrm{H} & -1.554627 & -1.574083 & 1.519223 \\ \mathrm{H} & -5.095705 & 2.345037 & 1.746729 \\ \mathrm{H} & -4.765887 & 2.743392 & 0.033481 \\ \mathrm{H} & 0.759742 & -2.852639 & 1.982131 \\ \mathrm{H} & 2.841644 & 1.857361 & 1.925336 \\ \mathrm{H} & 3.616671 & 0.050143 & 3.341733 \\ \mathrm{H} & 3.460006 & -1.916938 & 4.440885 \\ \mathrm{H} & 3.399268 & -3.213439 & 3.231144 \\ \mathrm{H} & 1.091797 & 0.543377 & -2.266606 \\ \mathrm{H} & 4.275095 & 3.761153 & -4.240894 \\ \mathrm{H} & -0.270200 & 4.082700 & -4.958800 \\ \mathrm{H} & -0.389400 & -2.199101 & -5.642800 \\ \mathrm{H} & -5.190900 & -2.322200 & -3.064100 \\ \mathrm{H} & -5.325797 & 1.123603 & 0.465900 \\ \mathrm{H} & -3.352102 & -5.417097 & 6.393200 \\ \mathrm{H} & 1.839001 & 1.274603 & 6.019600 \\ \mathrm{H} & -7.029002 & -2.820794 & 3.066701 \\ \mathrm{O} & 0.271945 & -0.030571 & -2.247599 \\ \mathrm{O} & 1.640362 & -1.347588 & -0.295384 \\ & & & \end{array}$

\section{TS3 $_{1}$}

$\begin{array}{llll}\mathrm{C} & -0.550650 & 3.635636 & -4.011807 \\ \mathrm{~N} & -0.747077 & 4.356572 & -2.847857 \\ \mathrm{C} & -0.695989 & 2.322103 & -3.656326 \\ \mathrm{C} & -1.004927 & 3.487154 & -1.846011 \\ \mathrm{~N} & -0.974152 & 2.245166 & -2.306475 \\ \mathrm{C} & 0.673129 & -2.155791 & -5.356832 \\ \mathrm{C} & 0.977557 & -3.246624 & -4.340676 \\ \mathrm{O} & 1.001786 & -4.439350 & -4.666741 \\ \mathrm{~N} & 1.220743 & -2.826120 & -3.077647 \\ \mathrm{C} & 3.796630 & 2.924929 & -3.745990 \\ \mathrm{~N} & 4.310629 & 2.329798 & -2.610386 \\ \mathrm{C} & 2.639057 & 2.236808 & -4.020528 \\ \mathrm{C} & 3.464145 & 1.328869 & -2.244658 \\ \mathrm{~N} & 2.442994 & 1.250597 & -3.076706 \\ \mathrm{C} & -4.211435 & -2.062758 & -2.686434 \\ \mathrm{~N} & -3.112047 & -2.870039 & -2.903254 \\ \mathrm{C} & -3.737680 & -0.968131 & -2.008302 \\ \mathrm{C} & -2.026325 & -2.265358 & -2.381897 \\ \mathrm{~N} & -2.372584 & -1.109578 & -1.831562 \\ \mathrm{~N} & -2.729640 & -4.816826 & 6.921812 \\ \mathrm{C} & -1.474331 & -4.573807 & 6.494274 \\ \mathrm{~N} & -0.996874 & -5.191994 & 5.415020 \\ \mathrm{~N} & -0.691501 & -3.739234 & 7.176792 \\ \mathrm{C} & 1.071340 & 1.009325 & 5.288455 \\ \mathrm{~N} & 0.642020 & -0.294720 & 5.139370 \\ \mathrm{C} & 0.357195 & 1.753376 & 4.379221 \\ \mathrm{C} & -0.284279 & -0.352166 & 4.177917 \\ \mathrm{~N} & -0.475870 & 0.879628 & 3.704644 \\ \mathrm{C} & -5.941538 & -2.806071 & 3.008868 \\ \mathrm{C} & -5.298502 & -2.777223 & 1.764561 \\ \mathrm{C} & -5.175334 & -2.815173 & 4.181974 \\ \mathrm{C} & -3.901923 & -2.771357 & 1.683828\end{array}$

\begin{tabular}{|c|c|c|c|}
\hline & -3.778925 & 1921 & \\
\hline $\mathrm{C}$ & 273 & -2.801307 & \\
\hline & 3419 & 322 & \\
\hline & & & \\
\hline & -3.156460 & 1.398499 & 8000 \\
\hline & & & \\
\hline & 5730 & & \\
\hline & 3407 & & \\
\hline 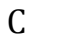 & 2.341896 & -1.968021 & \\
\hline $\mathrm{C}$ & & & \\
\hline & & & \\
\hline & & -0.3 & \\
\hline & & & \\
\hline & 2.640221 & -0.5 & \\
\hline C & & -2.6 & \\
\hline 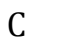 & & -3.3 & \\
\hline 0 & & & \\
\hline 0 & & -2.7 & \\
\hline U & -0.2 & -2.4 & \\
\hline 0 & & & \\
\hline $\mathrm{H}$ & 26 & & \\
\hline $\mathrm{H}$ & & & \\
\hline $\mathrm{H}$ & -1.2 & & \\
\hline $\mathrm{H}$ & & -2. & \\
\hline $\mathrm{H}$ & 0.88 & -1 & \\
\hline $\mathrm{H}_{\mathrm{H}}$ & 1.4 & & \\
\hline$H_{2}$ & & & \\
\hline $\mathrm{H}$ & & & \\
\hline $\mathrm{H}$ & & & \\
\hline H & 3.6 & & \\
\hline H & & & -1 \\
\hline $\mathrm{H}$ & & & \\
\hline $\mathrm{H}$ & & & \\
\hline $\mathrm{H}$ & -3.1 & & \\
\hline $\mathrm{H}$ & -0.0 & & \\
\hline 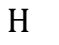 & & & \\
\hline $\mathrm{H}$ & & & \\
\hline $\mathrm{H}$ & & & \\
\hline $\mathrm{H}_{\text {- }}$ & & & \\
\hline $\mathrm{H}$ & & & \\
\hline $\mathrm{H}$ & & & \\
\hline 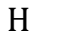 & & & \\
\hline $\mathrm{H}$ & $-5 . \varepsilon$ & & \\
\hline $\mathrm{H}$ & -5.6 & $-2 . \varepsilon$ & \\
\hline $\mathrm{H}$ & -3.4 & & \\
\hline 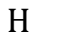 & & & \\
\hline $\mathrm{U}$ & & & \\
\hline $\mathrm{H}$ & -4.9 & & \\
\hline $\mathrm{H}$ & & & \\
\hline $\mathrm{H}$ & & -3.6 & \\
\hline $\mathrm{H}$ & & & \\
\hline $\mathrm{H}$ & & & \\
\hline H & & & \\
\hline $\mathrm{H}$ & 3.702974 & -3.43 & 3.61010 \\
\hline $\mathrm{H}$ & & & 2.1 \\
\hline $\mathrm{H}$ & & & \\
\hline & & & \\
\hline $\mathrm{H}$ & & 00 & \\
\hline & -5.190901 & -2.322199 & $-3.00^{2}$ \\
\hline
\end{tabular}




$\begin{array}{cccc}\mathrm{H} & -5.325798 & 1.123605 & 0.465899 \\ \mathrm{H} & -3.352104 & -5.417096 & 6.393201 \\ \mathrm{H} & 1.839000 & 1.274604 & 6.019603 \\ \mathrm{H} & -7.029003 & -2.820792 & 3.066701 \\ \mathrm{O} & 0.153963 & -0.245052 & -2.597563 \\ \mathrm{O} & 2.006745 & -0.938515 & -0.497874\end{array}$

\section{$\mathrm{Br}_{\mathrm{C1}}$ with His200 removed}

\begin{tabular}{|c|c|c|c|}
\hline $\mathrm{C}$ & -0.595765 & 3.623862 & -4.024123 \\
\hline $\mathrm{N}$ & -1.153585 & 4.376420 & -3.011288 \\
\hline C & -0.590882 & 2.330283 & -3.548782 \\
\hline $\mathrm{C}$ & -1.460124 & 3.555626 & -1.982553 \\
\hline $\mathrm{N}$ & -1.131632 & 2.304510 & -2.272818 \\
\hline $\mathrm{C}$ & -0.636567 & -2.015911 & -4.587514 \\
\hline $\mathrm{C}$ & -0.363892 & -3.286067 & -3.802281 \\
\hline 0 & -1.055136 & -4.297746 & -3.950134 \\
\hline $\mathrm{N}$ & 0.685955 & -3.253680 & -2.944493 \\
\hline $\mathrm{C}$ & -4.279611 & -1.937082 & -2.622210 \\
\hline $\mathrm{N}$ & -3.246324 & -2.774404 & -2.265221 \\
\hline $\mathrm{C}$ & -3.864649 & -0.671615 & -2.271052 \\
\hline C & -2.262636 & -2.028656 & -1.720705 \\
\hline $\mathrm{N}$ & -2.602497 & -0.747073 & -1.711 \\
\hline $\mathrm{N}$ & -2.735 & -4.66 & 6.68 \\
\hline $\mathrm{C}$ & -1.55 & -4.454 & 6.072979 \\
\hline $\mathrm{N}$ & -1.286803 & -5.041780 & 4.907795 \\
\hline $\mathrm{N}$ & -0.65 & -3.63 & 6.62 \\
\hline $\mathrm{C}$ & 1.051 & 1.111358 & 5.289943 \\
\hline $\mathrm{N}$ & 0.67 & -0.157388 & 4.905276 \\
\hline $\mathrm{C}$ & 0.253129 & 1.984758 & 4.593898 \\
\hline $\mathrm{C}$ & -0.315 & -0.071307 & 4.009954 \\
\hline $\mathrm{N}$ & -0.582204 & 1.218618 & 3.805207 \\
\hline $\mathrm{C}$ & -5.96 & -2.627 & \\
\hline $\mathrm{C}$ & -5.522861 & -2.113420 & 1.659856 \\
\hline $\mathrm{C}$ & -5.026243 & -2.895981 & 3.889750 \\
\hline $\mathrm{C}$ & -4.159421 & -1.887628 & 1.422648 \\
\hline $\mathrm{C}$ & -3.665786 & -2.673131 & 3.664831 \\
\hline $\mathrm{C}$ & -3.220319 & -2.184656 & 2.424410 \\
\hline 0 & -1.874827 & -2.036446 & 2.270364 \\
\hline $\mathrm{C}$ & -4.930479 & 1.713422 & 1.302679 \\
\hline $\mathrm{C}$ & -3.410388 & 1.716465 & 1.276301 \\
\hline 0 & -2.878560 & 1.734584 & 0.104417 \\
\hline 0 & -2.778018 & 1.736642 & 2.351577 \\
\hline $\mathrm{Fe}$ & -1.383203 & 0.763032 & -0.7 \\
\hline $\mathrm{C}$ & 2.240069 & -1.625435 & 2.224225 \\
\hline $\mathrm{C}$ & 1.295802 & -1.851336 & 1.282890 \\
\hline $\mathrm{C}$ & 0.698360 & -0.744302 & 0.465383 \\
\hline $\mathrm{C}$ & 1.122772 & 0.681500 & 0.880635 \\
\hline $\mathrm{C}$ & 2.208911 & 0.841610 & 1.826590 \\
\hline $\mathrm{C}$ & 2.716681 & -0.254443 & 2.453476 \\
\hline $\mathrm{C}$ & 2.789563 & -2.756727 & 3.079452 \\
\hline $\mathrm{C}$ & 1.692356 & -3.335368 & 3.994475 \\
\hline 0 & 1.084851 & -4.358888 & 3.617137 \\
\hline 0 & 1.450958 & -2.703327 & 5.083351 \\
\hline 0 & -0.629959 & -0.804816 & 0.308930 \\
\hline 0 & 0.499483 & 1.620859 & 0.365061 \\
\hline $\mathrm{H}$ & -0.251010 & 1.421750 & -4.034029 \\
\hline
\end{tabular}

\begin{tabular}{cccc}
$\mathrm{H}$ & -1.316018 & 5.378544 & -3.033293 \\
$\mathrm{H}$ & -1.919046 & 3.879768 & -1.055167 \\
$\mathrm{H}$ & -0.063943 & -1.154411 & -4.223501 \\
$\mathrm{H}$ & -1.707982 & -1.785934 & -4.532708 \\
$\mathrm{H}$ & 0.866642 & -4.073803 & -2.371831 \\
$\mathrm{H}$ & 1.138291 & -2.385706 & -2.674387 \\
$\mathrm{H}$ & -4.380366 & 0.277034 & -2.366183 \\
$\mathrm{H}$ & -3.179584 & -3.769695 & -2.460482 \\
$\mathrm{H}$ & -1.335357 & -2.426701 & -1.333321 \\
$\mathrm{H}$ & -2.915959 & -4.271006 & 7.604690 \\
$\mathrm{H}$ & -0.401042 & -4.815652 & 4.399705 \\
$\mathrm{H}$ & -2.024157 & -5.514573 & 4.395766 \\
$\mathrm{H}$ & -0.820447 & -3.231799 & 7.534601 \\
$\mathrm{H}$ & 0.183893 & -3.324910 & 6.076841 \\
$\mathrm{H}$ & 0.207297 & 3.066759 & 4.594619 \\
$\mathrm{H}$ & 1.113609 & -1.081532 & 5.139917 \\
$\mathrm{H}$ & -1.344661 & 1.540037 & 3.154810 \\
$\mathrm{H}$ & -0.815425 & -0.904595 & 3.516639 \\
$\mathrm{H}$ & -6.240122 & -1.882840 & 0.870959 \\
$\mathrm{H}$ & -5.352514 & -3.281249 & 4.857434 \\
$\mathrm{H}$ & -3.825478 & -1.484364 & 0.467336 \\
$\mathrm{H}$ & -2.927345 & -2.869411 & 4.439122 \\
$\mathrm{H}$ & -1.577107 & -1.612009 & 1.412387 \\
$\mathrm{H}$ & -5.304149 & 1.319145 & 2.254114 \\
$\mathrm{H}$ & -5.289486 & 2.747443 & 1.183955 \\
$\mathrm{H}$ & 0.904525 & -2.850402 & 1.090788 \\
$\mathrm{H}$ & 2.555825 & 1.848438 & 2.053878 \\
$\mathrm{H}$ & 3.509371 & -0.119913 & 3.190927 \\
$\mathrm{H}$ & 3.620371 & -2.398257 & 3.699325 \\
$\mathrm{H}$ & 3.162378 & -3.563213 & 2.436383 \\
$\mathrm{H}$ & 1.351662 & 0.655069 & -1.941980 \\
$\mathrm{H}$ & -0.270200 & 4.082700 & -4.958800 \\
$\mathrm{H}$ & -0.389400 & -2.199100 & -5.642800 \\
$\mathrm{H}$ & -5.190900 & -2.322200 & -3.064101 \\
$\mathrm{H}$ & -5.325797 & 1.123604 & 0.465900 \\
& -3.352103 & -5.417098 & 6.393200 \\
$\mathrm{H}$ & 1.839001 & 1.274601 & 6.019602 \\
\hline & -7.029002 & -2.820794 & 3.066700 \\
\hline
\end{tabular}

\section{$\mathrm{Br}_{\mathrm{C1}}$ with His200 removed}

$\begin{array}{lrrr}\mathrm{C} & -0.710485 & 3.513171 & -4.140931 \\ \mathrm{~N} & -2.023436 & 3.687915 & -3.754963 \\ \mathrm{C} & -0.212986 & 2.548670 & -3.294061 \\ \mathrm{C} & -2.280872 & 2.857219 & -2.718160 \\ \mathrm{~N} & -1.204216 & 2.146949 & -2.415732 \\ \mathrm{C} & -0.822834 & -2.222338 & -4.634308 \\ \mathrm{C} & -0.571214 & -3.589658 & -4.020510 \\ \mathrm{O} & -1.354368 & -4.529890 & -4.188117 \\ \mathrm{~N} & 0.568949 & -3.719639 & -3.301892 \\ \mathrm{C} & -4.247009 & -1.991687 & -2.638426 \\ \mathrm{~N} & -3.241045 & -2.883166 & -2.330605 \\ \mathrm{C} & -3.761087 & -0.755715 & -2.275332 \\ \mathrm{C} & -2.204640 & -2.195279 & -1.810239 \\ \mathrm{~N} & -2.482635 & -0.899261 & -1.764487\end{array}$




\begin{tabular}{|c|c|c|c|}
\hline & 07 & -4.739562 & \\
\hline & -1.489183 & & \\
\hline & 5655 & & \\
\hline & 9076 & 2031 & \\
\hline & 1.077940 & 1.083257 & 078 \\
\hline & 0.646632 & -0.199040 & \\
\hline & 0.34 & 917354 & \\
\hline & -0.309768 & & \\
\hline & -0.501987 & 550 & \\
\hline & -5.961662 & -2.682711 & \\
\hline & -5.43 & & \\
\hline & & & \\
\hline & -4.059024 & -2.159938 & 28 \\
\hline & -3.722405 & -2.731718 & \\
\hline & -3.2 & & \\
\hline & & & \\
\hline & -4.8 & & \\
\hline & -3.3 & & \\
\hline & -2.8 & & \\
\hline & -2.6 & & \\
\hline $\mathrm{Fe}$ & -1.2 & 90 & \\
\hline & & & \\
\hline & 1.1 & & \\
\hline & & 91 & \\
\hline & 1.4 & -0. & \\
\hline & 2.6 & & \\
\hline & 3.01 & -0.4 & \\
\hline & & & \\
\hline & & & \\
\hline & & -4 & \\
\hline & & & \\
\hline & -0.6 & & \\
\hline & & & \\
\hline & 0.7 & & 891 \\
\hline & -2.6 & & 773 \\
\hline & -3.2 & & 978 \\
\hline & & & \\
\hline & & & \\
\hline & & -4 & 670 \\
\hline & & & 428 \\
\hline & & & 468 \\
\hline & & & \\
\hline & -1 & -2 & 074 \\
\hline & -2.8 & & 612 \\
\hline & -0.3 & & 778 \\
\hline & & & \\
\hline & -0 & & \\
\hline & & -3. & \\
\hline & & & \\
\hline & & & 753 \\
\hline & & & \\
\hline & & & \\
\hline & & & \\
\hline & -5.5 & -3.1 & 5.000466 \\
\hline & & & 967 \\
\hline & & & \\
\hline & & & \\
\hline & -5.181167 & 1.261293 & 2.251658 \\
\hline & -5.099269 & 2.714884 & $1.2262 z$ \\
\hline
\end{tabular}

$\begin{array}{cccc}\mathrm{H} & 0.560119 & -2.897184 & 2.032354 \\ \mathrm{H} & 3.156652 & 1.218309 & 0.637265 \\ \mathrm{H} & 3.890965 & -0.175603 & 2.548306 \\ \mathrm{H} & 3.488913 & -1.888960 & 4.128383 \\ \mathrm{H} & 3.535548 & -3.276567 & 3.030370 \\ \mathrm{H} & 1.213965 & -0.156921 & -2.566580 \\ \mathrm{H} & -0.270200 & 4.082700 & -4.958800 \\ \mathrm{H} & -0.389400 & -2.199100 & -5.642799 \\ \mathrm{H} & -5.190900 & -2.322200 & -3.064100 \\ \mathrm{H} & -5.325796 & 1.123604 & 0.465901 \\ \mathrm{H} & -3.352102 & -5.417098 & 6.393201 \\ \mathrm{H} & 1.839002 & 1.274601 & 6.019603 \\ \mathrm{H} & -7.029001 & -2.820794 & 3.066701 \\ \mathrm{O} & 1.180802 & -1.021356 & -2.108850 \\ \mathrm{O} & 2.132355 & -0.816146 & -1.037711\end{array}$

\section{$\mathrm{Br}_{\mathrm{C} 2}$ with Tyr257 removed}

$\begin{array}{lccc}\mathrm{C} & -0.578596 & 3.615893 & -4.022081 \\ \mathrm{~N} & -1.070447 & 4.366326 & -2.971762 \\ \mathrm{C} & -0.599698 & 2.314357 & -3.571254 \\ \mathrm{C} & -1.368757 & 3.531078 & -1.950411 \\ \mathrm{~N} & -1.093030 & 2.277290 & -2.278657 \\ \mathrm{C} & -0.859029 & -2.083713 & -4.654153 \\ \mathrm{C} & -0.426207 & -3.246827 & -3.779309 \\ \mathrm{O} & -0.980851 & -4.348058 & -3.837304 \\ \mathrm{~N} & 0.633199 & -3.006476 & -2.968267 \\ \mathrm{C} & 3.616701 & 3.255492 & -3.543680 \\ \mathrm{~N} & 3.019718 & 3.914982 & -2.488389 \\ \mathrm{C} & 3.186651 & 1.952836 & -3.445608 \\ \mathrm{C} & 2.267697 & 3.021629 & -1.800583 \\ \mathrm{~N} & 2.350539 & 1.825357 & -2.355477 \\ \mathrm{C} & -4.280838 & -1.946722 & -2.603380 \\ \mathrm{~N} & -3.287295 & -2.801610 & -2.174495 \\ \mathrm{C} & -3.838660 & -0.683365 & -2.274741 \\ \mathrm{C} & -2.302311 & -2.063933 & -1.615930 \\ \mathrm{~N} & -2.602093 & -0.774955 & -1.661802 \\ \mathrm{~N} & -2.646950 & -4.808727 & 6.798058 \\ \mathrm{C} & -1.561179 & -4.430544 & 6.090457 \\ \mathrm{~N} & -1.297960 & -4.977322 & 4.904634 \\ \mathrm{~N} & -0.736743 & -3.505756 & 6.582807 \\ \mathrm{C} & 1.065892 & 1.141966 & 5.259486 \\ \mathrm{~N} & 0.714592 & -0.107976 & 4.791210 \\ \mathrm{C} & 0.271955 & 2.042122 & 4.587502 \\ \mathrm{C} & -0.241987 & 0.019091 & 3.869524 \\ \mathrm{~N} & -0.528109 & 1.312317 & 3.729320 \\ \mathrm{C} & -4.905922 & 1.849654 & 1.181699 \\ \mathrm{C} & -3.380542 & 1.778108 & 1.214864 \\ \mathrm{O} & -2.806320 & 1.763520 & 0.070455 \\ \mathrm{O} & -2.792302 & 1.773640 & 2.321535 \\ \mathrm{Fe} & -1.290581 & 0.705290 & -0.755189 \\ \mathrm{C} & 2.318059 & -1.632081 & 2.261937 \\ \mathrm{C} & 1.336925 & -1.847047 & 1.357172 \\ \mathrm{C} & 0.734342 & -0.744933 & 0.533021 \\ \mathrm{C} & 1.205102 & 0.676476 & 0.912079 \\ \mathrm{C} & 2.346950 & 0.821795 & 1.798165 \\ \mathrm{C} & 2.850591 & -0.273612 & 2.430201 \\ \mathrm{C} & 2.846961 & -2.763429 & 3.134945\end{array}$




$\begin{array}{crrr}\mathrm{C} & 1.728408 & -3.322699 & 4.031857 \\ \mathrm{O} & 1.143433 & -4.370516 & 3.684585 \\ \mathrm{O} & 1.434284 & -2.644517 & 5.081582 \\ \mathrm{O} & -0.591273 & -0.798519 & 0.428595 \\ \mathrm{O} & 0.577013 & 1.628873 & 0.427428 \\ \mathrm{H} & -0.287301 & 1.409196 & -4.078251 \\ \mathrm{H} & -1.211953 & 5.371405 & -2.971518 \\ \mathrm{H} & -1.788336 & 3.847131 & -1.002030 \\ \mathrm{H} & -0.568912 & -1.111846 & -4.235569 \\ \mathrm{H} & -1.947122 & -2.113926 & -4.785817 \\ \mathrm{H} & 0.900554 & -3.729815 & -2.306129 \\ \mathrm{H} & 0.901319 & -2.052165 & -2.734295 \\ \mathrm{H} & 3.421992 & 1.107081 & -4.083543 \\ \mathrm{H} & 3.134534 & 4.896530 & -2.257009 \\ \mathrm{H} & 1.677982 & 3.267418 & -0.924050 \\ \mathrm{H} & -4.318949 & 0.276990 & -2.424587 \\ \mathrm{H} & -3.242991 & -3.803631 & -2.335373 \\ \mathrm{H} & -1.400050 & -2.469871 & -1.178637 \\ \mathrm{H} & -2.938775 & -4.268552 & 7.606185 \\ \mathrm{H} & -0.386361 & -4.788380 & 4.428538 \\ \mathrm{H} & -1.897747 & -5.704118 & 4.529192 \\ \mathrm{H} & -0.885871 & -3.129584 & 7.512610 \\ \mathrm{H} & 0.116254 & -3.211393 & 6.052739 \\ \mathrm{H} & 0.207643 & 3.121485 & 4.648912 \\ \mathrm{H} & 1.141178 & -1.046539 & 5.023413 \\ \mathrm{H} & -1.293675 & 1.644230 & 3.082177 \\ \mathrm{H} & -0.691451 & -0.784741 & 3.297278 \\ \mathrm{H} & -5.331508 & 1.680833 & 2.177776 \\ \mathrm{H} & -5.204428 & 2.850829 & 0.835088 \\ \mathrm{H} & 0.908738 & -2.837420 & 1.198538 \\ \mathrm{H} & 2.736183 & 1.821783 & 1.985795 \\ \mathrm{H} & 3.681026 & -0.145969 & 3.126928 \\ \mathrm{H} & 3.666400 & -2.404976 & 3.770430 \\ \mathrm{H} & 3.230539 & -3.576269 & 2.506007 \\ \mathrm{H} & 1.388504 & 0.601311 & -1.992596 \\ \mathrm{H} & 4.275095 & 3.761153 & -4.240894 \\ \mathrm{H} & -0.270200 & 4.082700 & -4.958800 \\ \mathrm{H} & -0.389400 & -2.199100 & -5.642800 \\ \mathrm{H} & -5.190900 & -2.322200 & -3.064101 \\ \mathrm{H} & -5.325797 & 1.123604 & 0.465900 \\ \mathrm{H} & -3.352103 & -5.417098 & 6.393200 \\ \mathrm{H} & 1.839001 & 1.274601 & 6.019602 \\ \mathrm{O} & 0.719360 & -0.169948 & -1.805315 \\ \mathrm{O} & 1.422468 & -0.968661 & -0.810197\end{array}$

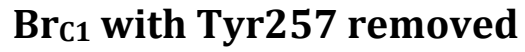

$\begin{array}{lrrr}\mathrm{C} & -0.604259 & 3.590344 & -4.045110 \\ \mathrm{~N} & -0.974742 & 4.310066 & -2.924457 \\ \mathrm{C} & -0.733942 & 2.270256 & -3.679674 \\ \mathrm{C} & -1.311450 & 3.437700 & -1.947275 \\ \mathrm{~N} & -1.170058 & 2.189759 & -2.369269 \\ \mathrm{C} & -0.717405 & -2.077051 & -4.600542 \\ \mathrm{C} & -0.308605 & -3.316523 & -3.821922 \\ \mathrm{O} & -0.861553 & -4.404081 & -4.009124 \\ \mathrm{~N} & 0.708101 & -3.155029 & -2.940557 \\ \mathrm{C} & 3.563087 & 3.181144 & -3.664715 \\ \mathrm{~N} & 3.024001 & 3.645100 & -2.481388\end{array}$

\begin{tabular}{|c|c|c|c|}
\hline & & & \\
\hline & & & \\
\hline & & 828 & \\
\hline & -4.253073 & .977010 & 26251 \\
\hline & -3.283275 & -2.869337 & 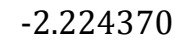 \\
\hline & -3.749790 & 284 & \\
\hline & -2.254848 & -2.172943 & \\
\hline & -2.499935 & -0.871621 & \\
\hline & -2.688576 & -4.845510 & \\
\hline & -1.52 & -4.4 & \\
\hline & & $-5 . c$ & \\
\hline & -0.7 & -3.592783 & \\
\hline & 1.055192 & 1.133283 & \\
\hline & & & \\
\hline & 0.31 & 2.0 & \\
\hline & -0.3 & & \\
\hline & -0.5 & & \\
\hline & -4.8 & 501 & \\
\hline & -3.3 & & \\
\hline & -2.7 & 1.5 & \\
\hline 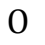 & -2.74 & & \\
\hline 10 & -1.2 & & \\
\hline C & 2.2 & -1.7 & \\
\hline $\mathrm{C}$ & 0.9 & -2. & \\
\hline 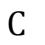 & 0.36 & -1. & \\
\hline & & & \\
\hline C & 2.4 & & \\
\hline$C$ & 2.9 & -0.5 & \\
\hline & 2.8 & -2 & \\
\hline $\mathrm{C}$ & & & \\
\hline 0 & $1.3^{7}$ & -4.5 & \\
\hline 0 & & & \\
\hline 0 & -0.8 & -1 & \\
\hline 0 & 0.4 & 63 & 0.1 \\
\hline H & & & \\
\hline $\mathrm{H}$ & & & \\
\hline & & & \\
\hline & -0.2 & -1 & \\
\hline $\mathrm{H}$ & -1.8 & & \\
\hline $\mathrm{H}$ & & -3.5 & -2.3 \\
\hline $\mathrm{H}$ & & & \\
\hline & & & \\
\hline H & & & \\
\hline $\mathrm{H}$ & & & \\
\hline $\mathrm{H}$ & & & -2 \\
\hline & & & \\
\hline & & & \\
\hline $\mathrm{H}$ & -3.0 & -4.3 & \\
\hline$\Pi$ & -0.1 & -4.8 & 4.7 \\
\hline $\mathrm{H}$ & & & \\
\hline$H$ & & & \\
\hline & & -3.2 & \\
\hline 11 & & & 11 \\
\hline $\mathrm{H}$ & & -1.0 & 5.22865 \\
\hline$M$ & & & \\
\hline$H_{H} \quad-\quad$ & & & \\
\hline & & & \\
\hline $\mathrm{H}$ & -5.140987 & 2.823958 & 0.93003 \\
\hline & 0434145 & -2.978968 & 010 \\
\hline
\end{tabular}




$\begin{array}{crcc} & & & \\ \mathrm{H} & 2.972170 & 1.238971 & 0.982988 \\ \mathrm{H} & 3.832916 & -0.334378 & 2.693617 \\ \mathrm{H} & 3.561359 & -2.159132 & 4.113681 \\ \mathrm{H} & 3.384919 & -3.510102 & 2.976224 \\ \mathrm{H} & 1.306690 & 0.375130 & -2.358027 \\ \mathrm{H} & 4.275095 & 3.761153 & -4.240894 \\ \mathrm{H} & -0.270200 & 4.082700 & -4.958800 \\ \mathrm{H} & -0.389400 & -2.199100 & -5.642799 \\ \mathrm{H} & -5.190900 & -2.322200 & -3.064100 \\ \mathrm{H} & -5.325796 & 1.123604 & 0.465901 \\ \mathrm{H} & -3.352102 & -5.417098 & 6.393201 \\ \mathrm{H} & 1.839002 & 1.274601 & 6.019603 \\ \mathrm{O} & 0.840888 & -0.432150 & -1.899505 \\ \mathrm{O} & 1.781164 & -0.640810 & -0.818709\end{array}$

\section{TS3 ${ }_{c 2}$ with Tyr257 removed}

$\begin{array}{lccc}\mathrm{C} & -0.509338 & 3.628865 & -3.997326 \\ \mathrm{~N} & -0.830316 & 4.364479 & -2.868588 \\ \mathrm{C} & -0.550501 & 2.318096 & -3.595918 \\ \mathrm{C} & -1.062202 & 3.509805 & -1.848755 \\ \mathrm{~N} & -0.896470 & 2.259493 & -2.257331 \\ \mathrm{C} & -1.087494 & -2.485095 & -4.840002 \\ \mathrm{C} & -0.421280 & -3.508566 & -3.934117 \\ \mathrm{O} & -0.731742 & -4.705678 & -3.952148 \\ \mathrm{~N} & 0.554212 & -3.010911 & -3.132789 \\ \mathrm{C} & 3.660196 & 3.064410 & -3.678929 \\ \mathrm{~N} & 3.252594 & 3.323010 & -2.385760 \\ \mathrm{C} & 3.127084 & 1.830412 & -3.981786 \\ \mathrm{C} & 2.513788 & 2.263079 & -1.961407 \\ \mathrm{~N} & 2.417014 & 1.343606 & -2.902900 \\ \mathrm{C} & -4.255788 & -2.061953 & -2.569143 \\ \mathrm{~N} & -3.319496 & -3.016588 & -2.224354 \\ \mathrm{C} & -3.720022 & -0.864009 & -2.156255 \\ \mathrm{C} & -2.269599 & -2.400200 & -1.642870 \\ \mathrm{~N} & -2.478454 & -1.092998 & -1.587156 \\ \mathrm{~N} & -2.932523 & -4.500055 & 6.513073 \\ \mathrm{C} & -1.643154 & -4.286508 & 6.192717 \\ \mathrm{~N} & -0.833092 & -5.313357 & 5.952126 \\ \mathrm{~N} & -1.156490 & -3.041673 & 6.160221 \\ \mathrm{C} & 1.076505 & 1.197957 & 5.235869 \\ \mathrm{~N} & 0.738164 & -0.011564 & 4.657196 \\ \mathrm{C} & 0.294201 & 2.146811 & 4.617225 \\ \mathrm{C} & -0.195840 & 0.187532 & 3.725379 \\ \mathrm{~N} & -0.486540 & 1.486600 & 3.685845 \\ \mathrm{C} & -4.724327 & 2.027123 & 0.687483 \\ \mathrm{C} & -3.224170 & 1.724961 & 0.828007 \\ \mathrm{O} & -2.643056 & 1.356433 & -0.257515 \\ \mathrm{O} & -2.668089 & 1.881612 & 1.936258 \\ \mathrm{Fe} & -1.027484 & 0.432691 & -1.126889 \\ \mathrm{C} & 2.202028 & -2.035784 & 2.217097 \\ \mathrm{C} & 1.303211 & -1.618981 & 1.281681 \\ \mathrm{C} & 1.270780 & -0.199368 & 0.709965 \\ \mathrm{C} & 2.117124 & 0.822016 & 1.569928 \\ \mathrm{C} & 3.138247 & 0.242691 & 2.454353 \\ \mathrm{C} & 3.155601 & -1.080647 & 2.763212 \\ \mathrm{C} & 2.214940 & -3.448673 & 2.731767 \\ \mathrm{C} & 1.616647 & -3.649163 & 4.143205 \\ & & & \end{array}$

\begin{tabular}{cccc} 
& & & \\
$\mathrm{O}$ & 1.468549 & -4.832659 & 4.519429 \\
$\mathrm{O}$ & 1.283692 & -2.626296 & 4.831322 \\
$\mathrm{O}$ & 0.011413 & 0.259820 & 0.448245 \\
$\mathrm{O}$ & 1.972231 & 2.023948 & 1.430432 \\
$\mathrm{H}$ & -0.343998 & 1.412351 & -4.149146 \\
$\mathrm{H}$ & -0.903119 & 5.375829 & -2.812517 \\
$\mathrm{H}$ & -1.350630 & 3.812813 & -0.848777 \\
$\mathrm{H}$ & -1.341065 & -1.570181 & -4.286128 \\
$\mathrm{H}$ & -1.985785 & -2.922218 & -5.289999 \\
$\mathrm{H}$ & 0.989402 & -3.655869 & -2.477888 \\
$\mathrm{H}$ & 0.543057 & -2.002538 & -2.886567 \\
$\mathrm{H}$ & 3.216554 & 1.265991 & -4.905462 \\
$\mathrm{H}$ & 3.495452 & 4.139117 & -1.833902 \\
$\mathrm{H}$ & 2.065031 & 2.216123 & -0.973497 \\
$\mathrm{H}$ & -4.123654 & 0.139465 & -2.215140 \\
$\mathrm{H}$ & -3.375695 & -4.011366 & -2.425505 \\
$\mathrm{H}$ & -1.381797 & -2.915442 & -1.300304 \\
$\mathrm{H}$ & -3.564375 & -3.725054 & 6.682384 \\
$\mathrm{H}$ & 0.110026 & -5.160968 & 5.526208 \\
$\mathrm{H}$ & -1.194851 & -6.261303 & 5.973440 \\
$\mathrm{H}$ & -1.786219 & -2.249516 & 6.226188 \\
$\mathrm{H}$ & -0.193995 & -2.874687 & 5.803500 \\
$\mathrm{H}$ & 0.229827 & 3.218086 & 4.763167 \\
$\mathrm{H}$ & 1.150085 & -0.964930 & 4.807460 \\
$\mathrm{H}$ & -1.184475 & 1.857634 & 3.004342 \\
$\mathrm{H}$ & -0.624997 & -0.568117 & 3.079183 \\
$\mathrm{H}$ & -5.112846 & 2.489192 & 1.603099 \\
$\mathrm{H}$ & -4.876239 & 2.715026 & -0.156194 \\
$\mathrm{H}$ & 0.604516 & -2.300480 & 0.797531 \\
$\mathrm{H}$ & 3.810151 & 0.948620 & 2.941661 \\
$\mathrm{H}$ & 3.891137 & -1.453945 & 3.477772 \\
$\mathrm{H}$ & 3.246171 & -3.834853 & 2.759633 \\
$\mathrm{H}$ & 1.660299 & -4.115035 & 2.058554 \\
$\mathrm{H}$ & 0.970592 & 0.130565 & -2.563320 \\
$\mathrm{H}$ & 4.275095 & 3.761153 & -4.240894 \\
$\mathrm{H}$ & -0.270200 & 4.082700 & -4.958800 \\
$\mathrm{H}$ & -0.389400 & -2.199100 & -5.642800 \\
$\mathrm{H}$ & -5.190900 & -2.322200 & -3.064101 \\
$\mathrm{H}$ & -5.325796 & 1.123604 & 0.465900 \\
$\mathrm{H}$ & -3.352103 & -5.417098 & 6.393199 \\
$\mathrm{H}$ & 1.839002 & 1.274600 & 6.019602 \\
$\mathrm{O}$ & 0.098922 & -0.324269 & -2.416495 \\
$\mathrm{O}$ & 2.112828 & -0.498293 & -0.323843 \\
& & & \\
\hline
\end{tabular}

